Appl. Set-Valued Anal. Optim. 1 (2019), No. 2, pp. 149-169

Available online at http://asvao.biemdas.com

https://doi.org/10.23952/asvao.1.2019.2.05

\title{
A HYBRID ITERATIVE ALGORITHM FOR A SPLIT MIXED EQUILIBRIUM PROBLEM AND A HIERARCHICAL FIXED POINT PROBLEM
}

\author{
SHAMSHAD HUSAIN*, NISHA SINGH \\ Department of Applied Mathematics, Aligarh Muslim University, Aligarh, India
}

\begin{abstract}
In this paper, we suggest and analyze a hybrid algorithm for finding a common solution of a split mixed equilibrium problem and a hierarcchical fixed point problem for a finite family of nonexpansive mappings. We prove the strong convergence of the iterative method under some mild conditions and derive some applications. Finally, we give a numerical example to justify the main results.
\end{abstract}

Keywords. Hierarchical fixed point problem; Split mixed equilibrium problem; Nonexpansive mapping; Strong convergence.

2010 Mathematics Subject Classification. 47H05, 47H09, $49 \mathrm{~J} 40$.

\section{INTRODUCTION}

Let $H_{1}$ and $H_{2}$ be real Hilbert spaces and their inner products and induced norms be denoted by $\langle.,$. and $\|$.$\| , respectively. Let C \subseteq H_{1}$ and $Q \subseteq H_{2}$ be nonempty, closed and convex sets. A mapping $S: C \rightarrow C$ is called nonexpansive if

$$
\|S x-S y\| \leq\|x-y\|, \quad \forall x, y \in C .
$$

We denote the set of fixed points of $S$ by $\operatorname{Fix}(S)$, i.e., $\operatorname{Fix}(S):=\{x \in C: S x=x\}$.

Let $U: C \rightarrow H$ be a nonexpansive mapping. The following problem is called a hierarchical fixed point problem: find $x^{*} \in F(T)$ such that

$$
\left\langle x^{*}-U x^{*}, x-x^{*}\right\rangle \geq 0, \quad \forall x \in F(T) .
$$

For solving a convex minimization problem, hybrid iterative methods are in the spotlight of optimization theory; see [1, 10,11, 12, 13, 14, 16, 19, 20] and the references therein. In 2001, Yamada [23] considered the following hybrid steepest-descent iterative method:

$$
x_{m+1}=S x_{m}-\mu \lambda_{m} T\left(S x_{m}\right),
$$

where $T$ is a $l$-Lipschitzian continuous and $\eta$-strongly monotone operator with $l>0, \eta>0$ and $0<$ $\mu<\frac{2 \eta}{l^{2}}$. Under some appropriate conditions, he proved that the sequence $\left\{x_{m}\right\}$ defined by the descent method converges strongly to the unique solution of the variational inequality

$$
\left\langle T\left(x^{*}\right), x-x^{*}\right\rangle \geq 0, \quad \forall x \in F i x(S) .
$$

\footnotetext{
${ }^{*}$ Corresponding author.
}

E-mail addresses: s_husain68@yahoo.com (S. Husain), nishasingh096@gmail.com (N. Singh).

Received May 3, 2019; Accepted June 22, 2019.

(C)2019 Applied Set-Valued Analysis and Optimization 
In 2014, Zhang and Yang [24] proposed an explicit iterative algorithm based on the viscosity method for finding a solution for a class of variational inequalities over the common fixed point set of a finite family of nonexpansive mappings.

Theorem 1.1. Let $H$ be a real Hilbert space and let $T: H \rightarrow H$ be l-Lipschitzian continuous and $\eta$ strongly monotone mapping with $l>0$ and $\eta>0$. Let $\left\{S_{j}\right\}_{j=1}^{M}$ be M-nonexpansive mappings such that $\Xi=\bigcap_{j=1}^{M} \operatorname{Fix}\left(S_{j}\right) \neq \emptyset$ and let $V$ be $\rho$-Lipschitzian continuous with $\rho>0$. For any point $x_{0} \in H$, define a sequence $\left\{x_{m}\right\}$ as:

$$
x_{m+1}=\alpha_{m} \gamma V\left(x_{m}\right)+\left(I-\alpha_{m} \mu T\right) S_{M}^{m} S_{M-1}^{m} \ldots S_{1}^{m} x_{m}, \forall m \geq 0,
$$

where $0<\gamma \rho<\tau$ with $\tau=\mu\left(2 \eta-\mu l^{2}\right), 0<\mu<\frac{2 \eta}{l^{2}}, S_{j}^{m}=\left(1-\varpi_{m}^{j}\right) I+\varpi_{m}^{j} S_{j}$ for $j=1,2, \ldots, M$ and $\varpi_{m}^{j} \in\left(\zeta_{1}, \zeta_{2}\right)$ for some $\zeta_{1}, \zeta_{2} \in(0,1)$. If $\lim _{m \rightarrow \infty} \alpha_{m}=0, \sum_{m=1}^{\infty} \alpha_{m}=\infty$ and $\lim _{k \rightarrow \infty}\left|\varpi_{m}^{k}-\varpi_{m}^{k+1}\right|=0$, $\forall m=1,2, \cdot N$, then the sequence $\left\{x_{m}\right\}$ converges strongly to the unique solution $x^{*} \in \Xi$ of the variational inequality:

$$
\left\langle(\mu T-\gamma V) x^{*}, x-x^{*}\right\rangle \geq 0, \forall x \in \bigcap_{j=1}^{M} F i x\left(S_{j}\right) .
$$

Now we consider the following hierarchical fixed point problem (HFPP) for a finite family of nonexpansive mappings $\left\{S_{j}\right\}_{j=1}^{M}: C \rightarrow C$ with $\bigcap_{j=1}^{M} F i x\left(S_{j}\right) \neq \emptyset$ with respect to another nonexpansive nonself mapping $U: C \rightarrow H_{1}$ : Find $x^{*} \in \bigcap_{j=1}^{M} \operatorname{Fix}\left(S_{j}\right)$ such that

$$
\left\langle x^{*}-U x^{*}, x^{*}-x\right\rangle \leq 0, \forall x \in \bigcap_{j=1}^{M} F i x\left(S_{j}\right) .
$$

The solution set of the HFPP (1.1) is denoted by $\Phi$.

If we set $S_{j}=S$ for $j=1,2, \ldots, M$, a self nonexpansive mapping on $C$, then the HFPP (1.1) reduces to the following HFPP which is considered and studied by Moudafi and Maingé [17]: Find $x^{*} \in$ Fix $(S)$ such that

$$
\left\langle x^{*}-U x^{*}, x^{*}-x\right\rangle \leq 0, \quad \forall x \in \operatorname{Fix}(S) .
$$

In 1994, Blum and Oettli [3] introduced and studied the equilibrium problem (EP), which is to find $x \in C$ such that

$$
F(x, y) \geq 0, \quad \forall y \in C .
$$

where $F: C \times C \rightarrow \mathbb{R}$ is a bifunction. The solution set of problem (1.3) is denoted by $\operatorname{Sol}(E P)$. Problem (1.1) contains many problems, such as, Nash Equilibria problems, complementarity problems, fixed point problems and variational inequality problems as special cases $[1,2,6,7,8,12,14,19,20]$.

Now, we introduce the following split mixed equilibrium problem (SMEP): Find $x^{*} \in C$ such that

$$
F\left(x^{*}, x\right)+\left\langle f x^{*}, x-x^{*}\right\rangle \geq 0, \quad \forall x \in C,
$$

and

$$
y^{*}=A x^{*} \in Q \text { which solves } G\left(y^{*}, y\right)+\left\langle g y^{*}, y-y^{*}\right\rangle \geq 0, \quad \forall y \in Q,
$$

where $F: C \times C \rightarrow \mathbb{R}$ and $G: Q \times Q \rightarrow \mathbb{R}$ are two bifunctions, $A: H_{1} \rightarrow H_{2}$ is a bounded linear operator and $f: H_{1} \rightarrow H_{1}$ and $g: H_{2} \rightarrow H_{2}$ be two nonlinear mappings. 
The solution set of mixed equilibrium problem (1.4) is denoted by $\operatorname{Sol}(M E P)$ and the solution set of the SMEP (1.4)-(1.5) is denoted by $\Omega$. If $f=g=0$ in the SMEP (1.4)-(1.5), then it is reduced to the following split equilibrium problem (SEP): Find $x^{*} \in C$ such that

$$
F\left(x^{*}, x\right) \geq 0, \quad \forall x \in C,
$$

and

$$
y^{*}=A x^{*} \in Q \text { which solves } G\left(y^{*}, y\right) \geq 0, \forall y \in Q .
$$

The SEP (1.6)-(1.7) was initially given by Moudafi [18] and further studied by Kazmi and Rizvi [15]. The solution set of SEP (1.6)-(1.7) is denoted by $\Omega_{1}$.

If $F=G=0$, then SMEP (1.4)-(1.5) is reduced to the split variational inequality problem (SVIP): Find $x^{*} \in C$ such that

$$
\left\langle f x^{*}, x-x^{*}\right\rangle \geq 0, \quad \forall x \in C,
$$

and

$$
y^{*}=A x^{*} \in Q \text { which solves }\left\langle g y^{*}, y-y^{*}\right\rangle \geq 0, \forall y \in Q .
$$

The SVIP (1.8)-(1.9) was introduced and studied by Censor, Gibali and Reich [5]. The solution set of $\operatorname{SVIP}(1.8)-(1.9)$ is denoted by $\Omega_{2}$.

Recently, Moudafi [18] considered the following split monotone variational inclusion problem (SMVIP): Find $x^{*} \in H_{1}$ such that

$$
0 \in f\left(x^{*}\right)+N\left(x^{*}\right)
$$

and

$$
y^{*}=A x^{*} \in H_{2} \text { which solves } 0 \in g\left(y^{*}\right)+P\left(y^{*}\right),
$$

where $N: H_{1} \rightarrow 2^{H_{1}}$ and $P: H_{2} \rightarrow 2^{H_{2}}$ are multi-valued maximal monotone mappings. The solution set of SMVIP (1.10)-(1.11) is denoted by $\Omega_{3}$.

We observe that the problems the SMEP (1.4)-(1.5), the SEP (1.6)-(1.7) and the SVIP (1.8)-(1.9) can be deduced from the SMVIP (1.10)-(1.11).

If $f=g=0$, then the SMVIP(1.10)-(1.11) is reduced to the following split null point problem (SNPP): Find $x^{*} \in H_{1}$ such that

$$
0 \in N\left(x^{*}\right)
$$

and

$$
y^{*}=A x^{*} \in H_{2} \text { which solves } 0 \in P\left(y^{*}\right) .
$$

Byrne, Gibali and Reich [4] studied the weak and strong convergence theorems of some iterative methods for the SNPP (1.12)-(1.13).

In 2017, Kazmi, Ali and Furkan [14] analyzed a Krasnoselski-Mann type iterative method to approximate a solution of a hierarchical fixed point problem (1.2) for nonexpansive mappings and split mixed equilibrium problem (1.4)-(1.5). They proved weak convergence theorems and also proposed a hybrid iterative method for split monotone variational inclusion problem (1.10)-(1.11) and hierarchical fixed point problem (1.1). They proved that the sequences generated by their proposed hybrid iterative method is strongly convergent in real Hilbert spaces. The weak and strong convergence are different in infinite dimensional Hilbert spaces and the strong convergence is usually more desirable than the weak convergence. To prove strong convergence of algorithms for the SMEP (1.4)-(1.5) and the HFPP (1.1) is a more general and interesting problem which motivates our work. 
In this paper, we introduce a hybrid iterative method for finding a common solution of split mixed equilibrium problem the SMEP (1.4)-(1.5) and hierarchical fixed point problem (1.1)) for a finite family of nonexpansive mappings. We prove a strong convergence theorem for the proposed iterative algorithm. We give some applications of the convergence results. We also have given a numerical example. The results and methods discussed in this paper extend and unify various known results in this field.

\section{PRELIMINARIES}

We recall some important concepts and results, which will be used later. Let the symbols $\rightarrow$ and $\rightarrow$ denote strong and weak convergence, respectively.

For every point $x \in H_{1}$, there exists a unique nearest point in $C$ denoted by $P_{C} x$ such that

$$
\left\|x-P_{C} x\right\| \leq\|x-y\|, \quad \forall y \in C .
$$

The mapping $P_{C}$ is called the metric projection of $H_{1}$ onto $C$. It is known that $P_{C}$ is nonexpansive and satisfies

$$
\left\langle x-y, P_{C} x-P_{C} y\right\rangle \geq\left\|P_{C} x-P_{C} y\right\|^{2}, \quad \forall x \in H_{1} .
$$

Further, $P_{C} x$ is characterized by the fact $P_{C} x \in C$ and

$$
\left\langle x-P_{C} x, y-P_{C} x\right\rangle \leq 0, \quad \forall y \in C,
$$

which implies that

$$
\|x-y\|^{2} \geq\left\|x-P_{C} x\right\|^{2}+\left\|y-P_{C} x\right\|^{2}, \quad \forall x \in H_{1}, y \in C .
$$

Lemma 2.1. For all $x, y \in H_{1}$, there holds the inequality

$$
\|x+y\|^{2} \leq\|x\|^{2}+2\langle y, x+y\rangle .
$$

Definition 2.1. A mapping $f: H_{1} \rightarrow H_{1}$ is said to be

(i) monotone if

$$
\langle f x-f y, x-y\rangle \geq 0, \quad \forall x, y \in H_{1} ;
$$

(ii) $\alpha$-inverse strongly monotone if there exists a constant $\alpha>0$ such that

$$
\langle f x-f y, x-y\rangle \geq \alpha\|f x-f y\|^{2}, \quad \forall x, y \in H_{1} ;
$$

(iii) $\beta$-Lipschitz continuous if there exists a constant $\beta>0$ such that

$$
\|f x-f y\| \leq \beta\|x-y\|, \quad \forall x, y \in H_{1} .
$$

(iv) $\varpi$-averaged if there exists $\varpi \in(0,1)$ such that $S=(1-\varpi) I+\varpi U$, where $I: H_{1} \rightarrow H_{1}$ is the identity mapping and $U: H_{1} \rightarrow H_{1}$ is nonexpansive.

If $f$ is $\alpha$-inverse strongly monotone mapping, then $f$ is monotone and $\frac{1}{\alpha}$-Lipschitz continuous.

Lemma 2.2. [9] Let $S$ be a nonexpansive mapping on $H_{1}$. Then $S$ is demiclosed at the origin, that is, $\left\{x_{m}\right\}$ converges weakly to $x \in H_{1}$ and $\left\{x_{m}-S x_{m}\right\}$ converges strongly to 0 , then $x \in$ Fix $(S)$.

Lemma 2.3. [2] Let $C \subset H_{1}$ be a nonempty, closed and convex set and let $S: C \rightarrow H_{1}$ be a nonexpansive mapping. Then Fix $(S)$ is closed and convex.

Assumpion 2.1. The bifunction $F: C \times C \rightarrow \mathbb{R}$ satisfies the following conditions 
(i) $F(x, x)=0, \quad \forall x \in C$;

(ii) $F$ is monotone, i.e., $F(x, y)+F(y, x) \leq 0, \quad \forall x \in C$;

(iii) For each $x, y, z \in C$, $\limsup _{t \rightarrow 0} F(t z+(1-t) x, y) \leq F(x, y)$;

(iv) For each $x \in C, y \rightarrow F(x, y)$ is convex and lower semicontinuous.

Lemma 2.4. [8] Let $C$ be a nonempty closed convex subset of $H_{1}$. Assume that $F: C \times C \rightarrow \mathbb{R}$ satisfying Assumption 2.1. Then, for all $r>0$ and for all $x \in H_{1}$, define the resolvent operator $S_{r}: H_{1} \rightarrow C$ by

$$
S_{r}(x)=\left\{z \in C: F(z, y)+\frac{1}{r}\langle y-z, z-x\rangle \geq 0, \quad \forall y \in C\right\}
$$

Then the following holds:

(i) for each $x \in H_{1}, S_{r}(x) \neq \emptyset$;

(ii) $S_{r}$ is single-valued;

(iii) $S_{r}$ is firmly nonexpansive, i.e.,

$$
\left\|S_{r} x-S_{r} y\right\|^{2} \leq\left\langle S_{r} x-S_{r} y, x-y\right\rangle, \forall x, y \in H_{1} ;
$$

(iv) $\operatorname{Fix}\left(S_{r}\right)=\operatorname{Sol}(E P)$;

(v) Fix $\left(S_{r}\right)$ is closed and convex.

Lemma 2.5. Let $F_{1}: C \times C \rightarrow \mathbb{R}$ be a bifunction with Assumption 2.1 and let $S_{r}$ be defined as in Lemma 2.4. Let $x, y \in H_{1}$ and $r_{1}, r_{2}>0$. Then

$$
\left\|S_{r_{2}}(y)-S_{r_{1}}(x)\right\| \leq\|y-x\|+\left|\frac{r_{2}-r_{1}}{r_{2}}\right|\left\|S_{r_{2}}(y)-y\right\| .
$$

Lemma 2.6. [23] Suppose that $\lambda \in(0,1)$ and $\mu>0$. Let $T: C \rightarrow C$ be a l-Lipschitzian continuous and $\eta$-strongly monotone mapping with $l>0$ and $\eta>0$. Define a mapping $S^{\lambda}: C \rightarrow H_{1}$ by

$$
S^{\lambda} x=S x-\lambda \mu T(S x), \quad \forall x \in C,
$$

where $S$ is a nonexpansive mapping on $C$. Then $S^{\lambda}$ is a contraction provided $\mu<\frac{2 \eta}{l^{2}}$, i.e.,

$$
\left\|S^{\lambda} x-S^{\lambda} y\right\| \leq(1-\lambda \tau)\|x-y\|, \forall x, y \in C,
$$

where $\tau=1-\sqrt{1-\mu\left(2 \eta-\mu l^{2}\right)}$.

Lemma 2.7. [21] Let $\left\{x_{m}\right\}$ and $\left\{y_{m}\right\}$ be bounded sequences in a Hilbert space $H$ and let $\left\{\beta_{m}\right\}$ be a sequence in $[0,1]$ with $0<\liminf _{m \rightarrow \infty} \beta_{m} \leq \limsup _{m \rightarrow \infty} \beta_{m}<1$. Suppose $x_{m+1}=\beta_{m} x_{m}+\left(1-\beta_{m}\right) y_{m}$ for all integers $m \geq 0$ and $\limsup _{m \rightarrow \infty}\left(\left\|y_{m+1}-y_{m}\right\|-\left\|x_{m+1}-x_{m}\right\|\right) \leq 0$. Then $\lim _{m \rightarrow \infty}\left\|y_{m}-x_{m}\right\|=0$.

Lemma 2.8. [22] Assume that $\left\{\delta_{m}\right\}$ is a sequence of nonnegative real numbers such that

$$
\delta_{m+1} \leq\left(1-\gamma_{m}\right) \delta_{m}+\alpha_{m},
$$

where $\left\{\gamma_{m}\right\}$ is a sequence in $(0,1)$ and $\alpha_{m}$ is a sequence such that

(i) $\sum_{m=1}^{\infty} \gamma_{m}=\infty$;

(ii) $\lim \sup _{m \rightarrow \infty} \frac{\alpha_{m}}{\gamma_{m}} \leq 0$ or $\sum_{m=1}^{\infty}\left|\alpha_{m}\right|<\infty$.

Then $\lim _{m \rightarrow \infty} \delta_{m}=0$.

Lemma 2.9. Let $\left\{S_{j}\right\}_{j=1}^{M}$ be averaged mappings with common fixed points. Then $\bigcap_{j=1}^{M} F i x\left(S_{j}\right)=$ $\operatorname{Fix}\left(S_{1} \ldots S_{M}\right)$. 


\section{MAIN RESULTS}

We prove a strong convergence theorem for the SMEP (1.3)-(1.4) and the HFPP (1.1) in this section.

Theorem 3.1. Let $H_{1}$ and $H_{2}$ be two real Hilbert spaces and let $C \subseteq H_{1}$ and $Q \subseteq H_{2}$ be nonempty, closed and convex subsets. Let $A: H_{1} \rightarrow H_{2}$ be a bounded linear operator with its adjoint operator $A^{*}$. Let $F: C \times C \rightarrow \mathbb{R}$ and $G: Q \times Q \rightarrow \mathbb{R}$ be bifunctions satisfying Assumption 2.1 and let $G$ be upper semicontinuous. Let mappings $f: C \rightarrow H_{1}$ and $g: Q \rightarrow H_{2}$ be $\theta_{1}$-inverse strongly monotone and $\theta_{2}$-inverse strongly monotone, respectively. Let $S_{j}: C \rightarrow C$ be a nonexpansive mapping for each $j=1,2, \ldots, M$. Let $V: C \rightarrow C$ be a l-lipschitzian continuous and $\eta$-strongly monotone mapping with $l>0$ and $\eta>0$ and let $U: C \rightarrow C$ be a $\tau$-lipschitzian continuous mapping with $\tau>0$. Let $0<\mu<\frac{2 \eta}{l^{2}}$ and $0<\rho \tau<\zeta$, where $\zeta=1-\sqrt{1-\mu\left(2 \eta-\mu l^{2}\right)}$. Assume that $\Gamma=\Omega \bigcap \Phi \neq \emptyset$. Let the iterative sequences $\left\{z_{m}\right\}$ and $\left\{x_{m}\right\}$ be generated via the hybrid iterative algorithm:

$$
\left\{\begin{array}{l}
x_{0} \in C \\
y_{m}=X\left(x_{m}\right) ; w_{m}=Y\left(A y_{m}\right) \\
z_{m}=y_{m}+\gamma A^{*}\left(w_{m}-A y_{m}\right) \\
x_{m+1}=\alpha_{m} \rho U\left(x_{m}\right)+\beta_{m} x_{m}+\left[\left(1-\beta_{m}\right) I-\alpha_{m} \mu V\right] S_{M}^{m} S_{M-1}^{m} \ldots S_{1}^{m} z_{m}, \forall m \geq 0
\end{array}\right.
$$

where $S_{j}^{m}=\left(1-\varpi_{m}^{j}\right) I+\varpi_{m}^{j} S_{j}, X=S_{r_{m}}^{F}\left(I-r_{m} f\right), Y=S_{r_{m}}^{G}\left(I-r_{m} g\right)$ and $\gamma \in\left(0, \frac{1}{\|A\|^{2}}\right), \varpi_{m}^{j} \in\left(\xi_{1}, \xi_{2}\right)$ for some $\xi_{1}, \xi_{2} \in(0,1),\left\{\alpha_{m}\right\}$ and $\left\{\beta_{m}\right\}$ are two real sequences in $(0,1)$ and $\left\{r_{m}\right\} \subset(0, \alpha)$, where $\alpha=2 \min \left\{\theta_{1}, \theta_{2}\right\}$, satisfy the conditions:

(i) $\lim _{m \rightarrow \infty} \alpha_{m}=0$ and $\sum_{m=1}^{\infty} \alpha_{m}=\infty$.

(ii) $0<\liminf _{m \rightarrow \infty} \beta_{m} \leq \limsup _{m \rightarrow \infty} \beta_{m}<1$.

(iii) $\lim _{m \rightarrow \infty} \inf r_{m}>0$.

(iv) $\lim _{m \rightarrow \infty}\left|\varpi_{m+1}^{j}-\varpi_{m}^{j}\right|=0$ for $j=1,2, \ldots, M$.

Then the sequence $\left\{x_{m}\right\}$ converges strongly to $\bar{p} \in \Gamma$.

Proof. We divide the proof into five steps.

Step 1. Since $f: C \rightarrow H_{1}$ is $\theta_{1}$-inverse strongly monotone, we have

$$
\begin{aligned}
\left\|\left(I-r_{m} f\right) x-\left(I-r_{m} f\right) y\right\|^{2} & =\left\|(x-y)-r_{m}(f x-f y)\right\|^{2} \\
& \leq\|x-y\|^{2}-r_{m}\left(2 \theta_{1}-r_{m}\right)\|f x-f y\|^{2} \\
& \leq\|x-y\|^{2},
\end{aligned}
$$

which shows that $\left(I-r_{m} f\right)$ is nonexpansive. Similarly, we can show that $\left(I-r_{m} g\right)$ is nonexpansive too. Hence $X=S_{r_{m}}^{F}\left(I-r_{m} f\right), Y=S_{r_{m}}^{G}\left(I-r_{m} g\right)$ are nonexpansive. Since $\Gamma \neq \emptyset$, it follows from Lemma 2.3 that $\operatorname{Fix}\left(S_{r_{m}}^{F}\left(I-r_{m} f\right)\right)$ and $\operatorname{Fix}\left(S_{r_{m}}^{G}\left(I-r_{m} g\right)\right)$ are closed and convex sets. So, $\Omega$ is closed and convex. Since $\Phi=\bigcap_{j=1}^{M} \operatorname{Fix}\left(S_{j}\right) \neq \emptyset$, we have hat $\Phi$ is closed and convex. Thus, $\Gamma$ is nonempty, closed and convex. Let $p \in \Gamma$ then $p \in \Omega$. Then $S_{r_{m}}^{F}\left(I-r_{m} f\right) p=p$ and $S_{r_{m}}^{G}\left(I-r_{m} g\right) A p=A p$. From (3.1), we have

$$
\begin{aligned}
\left\|y_{m}-p\right\|^{2} & \leq\left\|\left(x_{m}-p\right)-r_{m}\left(f x_{m}-f p\right)\right\|^{2} \\
& \leq\left\|x_{m}-p\right\|^{2}-r_{m}\left(2 \theta_{1}-r_{m}\right)\left\|f x_{m}-f p\right\|^{2} \\
& \leq\left\|x_{m}-p\right\|^{2}
\end{aligned}
$$


and

$$
\begin{aligned}
\left\|w_{m}-A p\right\|^{2} & \leq\left\|\left(A y_{m}-A p\right)-r_{m}\left(g A y_{m}-g A p\right)\right\|^{2} \\
& \leq\left\|A y_{m}-A p\right\|^{2}-r_{m}\left(2 \theta_{2}-r_{m}\right)\left\|g A y_{m}-g A p\right\|^{2} \\
& \leq\left\|A y_{m}-A p\right\|^{2} .
\end{aligned}
$$

From (3.1) and (3.2), we evaluate

$$
\begin{aligned}
\left\|z_{m}-p\right\|^{2} \leq & \left\|y_{m}-p\right\|^{2}+\left\|\gamma A^{*}\left(w_{m}-A y_{m}\right)\right\|^{2}+2 \gamma\left\langle y_{m}-p, A^{*}\left(w_{m}-A y_{m}\right)\right\rangle \\
\leq & \left\|y_{m}-p\right\|^{2}+\gamma^{2}\left\|A^{*}\right\|^{2}\left\|w_{m}-A y_{m}\right\|^{2} \\
& +2 \gamma\left\langle A\left(y_{m}-p\right)+\left(w_{m}-A y_{m}\right)-\left(w_{m}-A y_{m}\right), w_{m}-A y_{m}\right\rangle \\
= & \left\|y_{m}-p\right\|^{2}+\gamma^{2}\left\|A^{*}\right\|^{2}\left\|w_{m}-A y_{m}\right\|^{2} \\
& +\gamma\left[\left\|w_{m}-A p\right\|^{2}+\left\|w_{m}-A y_{m}\right\|^{2}-\left\|A y_{m}-A p\right\|^{2}-2\left\|w_{m}-A y_{m}\right\|^{2}\right] \\
\leq & \left\|y_{m}-p\right\|^{2}-\gamma\left(1-\gamma\left\|A^{*}\right\|^{2}\right)\left\|w_{m}-A y_{m}\right\|^{2} \\
\leq & \left\|x_{m}-p\right\|^{2}-\gamma\left(1-\gamma\left\|A^{*}\right\|^{2}\right)\left\|w_{m}-A y_{m}\right\|^{2} \\
\leq & \left\|x_{m}-p\right\|^{2} .
\end{aligned}
$$

Using Lemma 2.6, we get

$$
\begin{aligned}
\left\|x_{m+1}-p\right\| \leq & \| \alpha_{m}\left(\rho U\left(x_{m}\right)-\mu V(p)\right)+\beta_{m}\left(x_{m}-p\right)+\left[\left(1-\beta_{m}\right) I-\alpha_{m} \mu V\right] S_{M}^{m} S_{M-1}^{m} \ldots S_{1}^{m} z_{m} \\
& -\left[\left(1-\beta_{m}\right) I-\alpha_{m} \mu V\right] S_{M}^{m} S_{M-1}^{m} \ldots S_{1}^{m} p \| \\
\leq & \alpha_{m}\left\|\rho U\left(x_{m}\right)-\mu V(p)\right\|+\beta_{m}\left\|x_{m}-p\right\|+\|\left[\left(1-\beta_{m}\right) I-\alpha_{m} \mu V\right] S_{M}^{m} S_{M-1}^{m} \ldots S_{1}^{m} z_{m} \\
& -\left[\left(1-\beta_{m}\right) I-\alpha_{m} \mu V\right] S_{M}^{m} S_{m-1}^{m} \ldots S_{1}^{m} p \| \\
= & \alpha_{m}\left\|\rho U\left(x_{m}\right)-\mu V(p)\right\|+\beta_{m}\left\|x_{m}-p\right\|+\left(1-\beta_{m}\right) \|\left(I-\frac{\alpha_{m} \mu V}{1-\beta_{m}}\right) S_{M}^{m} S_{M-1}^{m} \ldots S_{1}^{m} z_{m} \\
& -\left(I-\frac{\alpha_{m} \mu V}{1-\beta_{m}}\right) S_{M}^{m} S_{M-1}^{m} \ldots S_{1}^{m} p \| \\
\leq & \alpha_{m}\left\|\rho U\left(x_{m}\right)-\mu V(p)\right\|+\beta_{m}\left\|x_{m}-p\right\|+\left(1-\beta_{m}\right)\left(1-\frac{\alpha_{m} \zeta}{1-\beta_{m}}\right)\left\|z_{m}-p\right\| \\
\leq & \alpha_{m}\left\|\rho U\left(x_{m}\right)-\mu V(p)\right\|+\beta_{m}\left\|x_{m}-p\right\|+\left(1-\beta_{m}-\alpha_{m} \zeta\right)\left\|x_{m}-p\right\| \\
\leq & \alpha_{m} \rho\left\|U\left(x_{m}\right)-U(p)\right\|+\alpha_{m}\|\rho U(p)-\mu V(p)\|+\left(1-\alpha_{m} \zeta\right)\left\|x_{m}-p\right\| \\
\leq & \alpha_{m} \rho \tau\left\|x_{m}-p\right\|+\alpha_{m}\|\rho U(p)-\mu V(p)\|+\left(1-\alpha_{m} \zeta\right)\left\|x_{m}-p\right\| \\
\leq & \left(1-\alpha_{m}(\zeta-\rho \tau)\right)\left\|x_{m}-p\right\|+\alpha_{m}\|\rho U(p)-\mu V(p)\| \\
\leq & \left(1-\alpha_{m}(\zeta-\rho \tau)\right)\left\|x_{m}-p\right\|+\alpha_{m}(\zeta-\rho \tau) \frac{\|\rho U(p)-\mu V(p)\|}{\zeta-\rho \tau} \\
\leq & \max \left\{\left\|x_{m}-p\right\|, \frac{\|\rho U(p)-\mu V(p)\|}{\zeta-\rho \tau}\right\} .
\end{aligned}
$$

By induction on $m$, we have

$$
\left\|x_{m}-p\right\| \leq \max \left\{\left\|x_{o}-p\right\|, \frac{\rho U(p)-\mu V(p) \|}{\zeta-\rho \tau}\right\}, m=1,2, \ldots
$$

Therefore, $\left\{x_{m}\right\}$ is bounded and further it follows that $\left\{y_{m}\right\},\left\{w_{m}\right\}$ and $\left\{z_{m}\right\}$ are also bounded. 
Step 2. We show that

$$
\begin{aligned}
\lim _{m \rightarrow \infty}\left\|x_{m+1}-x_{m}\right\|=0, & \lim _{m \rightarrow \infty}\left\|x_{m}-y_{m}\right\|=0, \\
\lim _{m \rightarrow \infty}\left\|x_{m}-z_{m}\right\|=0, & \lim _{m \rightarrow \infty}\left\|x_{m}-w_{m}\right\|=0 .
\end{aligned}
$$

Setting $x_{m+1}=\beta_{m} x_{m}+\left(1-\beta_{m}\right) w_{m}, \forall m \geq 1$, we have

$$
\begin{aligned}
w_{m+1}-w_{m}= & \frac{x_{m+2}-\beta_{m+1} x_{m+1}}{1-\beta_{m+1}}-\frac{x_{m+1}-\beta_{m} x_{m}}{1-\beta_{m}} \\
= & \frac{\alpha_{m+1} \rho U\left(x_{m+1}\right)+\left(\left(1-\beta_{m+1}\right) I-\alpha_{m+1} \mu V\right) S_{M}^{m+1} S_{M-1}^{m+1} \ldots S_{1}^{m+1} z_{m+1}}{1-\beta_{m+1}} \\
& -\frac{\alpha_{m} \rho U\left(x_{m}\right)+\left(\left(1-\beta_{m}\right) I-\alpha_{m} \mu V\right) S_{M}^{m} S_{M-1}^{m} \ldots S_{1}^{m} z_{m}}{1-\beta_{m}} \\
= & \frac{\alpha_{m+1}}{1-\beta_{m+1}}\left[\rho U\left(x_{m+1}\right)-\mu V\left(S_{M}^{m+1} S_{M-1}^{m+1} \ldots S_{1}^{m+1} z_{m+1}\right)\right] \\
& +\frac{\alpha_{m}}{1-\beta_{m}}\left[\mu V\left(S_{M}^{m} S_{M-1}^{m} \ldots S_{1}^{m} z_{m}\right)-\rho U\left(x_{m}\right)\right] \\
& +\left(S_{M}^{m+1} S_{M-1}^{m+1} \ldots S_{1}^{m+1} z_{m+1}-S_{M}^{m} S_{M-1}^{m} \ldots S_{1}^{m} z_{m}\right) \\
= & \frac{\alpha_{m+1}}{1-\beta_{m+1}}\left[\rho U\left(x_{m+1}\right)-\mu V\left(S_{M}^{m+1} S_{M-1}^{m+1} \ldots S_{1}^{m+1} z_{m+1}\right)\right] \\
& +\frac{\alpha_{m}}{1-\beta_{m}}\left[\mu V\left(S_{M}^{m} S_{M-1}^{m} \ldots S_{1}^{m} z_{m}\right)-\rho U\left(x_{m}\right)\right] \\
& +\left(S_{M}^{m+1} S_{M-1}^{m+1} \ldots S_{1}^{m+1} z_{m+1}-S_{M}^{m+1} S_{M-1}^{m+1} \ldots S_{1}^{m+1} z_{m}\right. \\
& \left.+S_{M}^{m+1} S_{M-1}^{m+1} \ldots S_{1}^{m+1} z_{m}-S_{M}^{m} S_{M-1}^{m} \ldots S_{1}^{m} z_{m}\right) .
\end{aligned}
$$

It follows

$$
\begin{aligned}
\left\|w_{m+1}-w_{m}\right\|-\left\|x_{m+1}-x_{m}\right\| \leq & \frac{\alpha_{m+1}}{1-\beta_{m+1}}\left[\rho\left\|U\left(x_{m+1}\right)\right\|+\mu\left\|V\left(S_{M}^{m+1} S_{M-1}^{m+1} \ldots S_{1}^{m+1} z_{m+1}\right)\right\|\right] \\
& +\frac{\alpha_{m}}{1-\beta_{m}}\left[\mu\left\|V\left(S_{M}^{m} S_{M-1}^{m} \ldots S_{1}^{m} z_{m}\right)\right\|+\rho\left\|U\left(x_{m}\right)\right\|\right] \\
& +\left\|S_{M}^{m+1} S_{M-1}^{m+1} \ldots S_{1}^{m+1} z_{m}-S_{M}^{m} S_{M-1}^{m} \ldots S_{1}^{m} z_{m}\right\| \\
& +\left\|z_{m+1}-z_{m}\right\|-\left\|x_{m+1}-x_{m}\right\| .
\end{aligned}
$$

From the definition of $S_{j}^{m}$, we have

$$
\begin{aligned}
\left\|S_{2}^{m} S_{1}^{m} z_{m}-S_{2}^{m-1} S_{1}^{m-1} z_{m}\right\| \leq & \left\|S_{2}^{m} S_{1}^{m} z_{m}-S_{2}^{m} S_{1}^{m-1} z_{m}\right\|+\left\|S_{2}^{m} S_{1}^{m-1} z_{m}-S_{2}^{m-1} s_{1}^{m-1} z_{m}\right\| \\
\leq & \left\|S_{1}^{m} z_{m}-S_{1}^{m-1} z_{m}\right\|+\left\|S_{2}^{m} S_{1}^{m-1} z_{m}-S_{2}^{m-1} S_{1}^{m-1} z_{m}\right\| \\
\leq & \left\|\left(1-\varpi_{m}^{1}\right) z_{m}+\varpi_{m}^{1} S_{1} z_{m}-\left(1-\varpi_{m-1}^{1}\right) z_{m}-\varpi_{m-1}^{1} S_{1} z_{m}\right\| \\
& +\|\left(1-\varpi_{m}^{2}\right) S_{1}^{m-1} z_{m}+\varpi_{m}^{2} S_{2} S_{1}^{m-1} z_{m} \\
& -\left(1-\varpi_{m-1}^{2}\right) S_{1}^{m-1} z_{m}-\varpi_{m-1}^{2} S_{2} S_{1}^{m-1} z_{m} \| \\
\leq & \left|\varpi_{m}^{1}-\varpi_{m-1}^{1}\right|\left(\left\|z_{m}\right\|+\left\|S_{1} z_{m}\right\|\right) \\
& +\left|\varpi_{m}^{2}-\varpi_{m-1}^{2}\right|\left(\left\|S_{1}^{m-1} z_{m}\right\|+\left\|S_{2} S_{1}^{m-1} z_{m}\right\|\right) .
\end{aligned}
$$


From (3.6), we have

$$
\begin{aligned}
& \left\|S_{3}^{m} S_{2}^{m} S_{1}^{m} z_{m}-S_{3}^{m-1} S_{2}^{m-1} S_{1}^{m-1} z_{m}\right\| \\
\leq & \left\|S_{3}^{m} S_{2}^{m} S_{1}^{m} z_{m}-S_{3}^{m} S_{2}^{m-1} S_{1}^{m-1} z_{m}\right\|+\left\|S_{3}^{m} S_{2}^{m-1} S_{1}^{m-1} z_{m}-S_{3}^{m-1} S_{2}^{m-1} S_{1}^{m-1} z_{m}\right\| \\
\leq & \left\|S_{2}^{m} S_{1}^{m} z_{m}-S_{2}^{m-1} S_{1}^{m-1} z_{m}\right\|+\|\left(1-\varpi_{m}^{3}\right) S_{2}^{m-1} S_{1}^{m-1} z_{m}+\varpi_{m}^{3} S_{3} S_{2}^{m-1} S_{1}^{m-1} z_{m} \\
& -\left(1-\varpi_{m-1}^{3}\right) S_{2}^{m-1} S_{1}^{m-1} z_{m}-\varpi_{m-1}^{3} S_{3} S_{2}^{m-1} S_{1}^{m-1} z_{m} \| \\
\leq & \left|\varpi_{m}^{1}-\varpi_{m-1}^{1}\right|\left(\left\|z_{m}\right\|+\left\|S_{1} z_{m}\right\|\right)+\left|\varpi_{m}^{2}-\varpi_{m-1}^{2}\right|\left(\left\|S_{1}^{m-1} z_{m}\right\|+\left\|S_{2} S_{1}^{m-1} z_{m}\right\|\right) \\
& +\left|\varpi_{m}^{3}-\varpi_{m-1}^{3}\right|\left(\left\|S_{2}^{m-1} S_{1}^{m-1} z_{m}\right\|+\left\|S_{3} S_{2}^{m-1} S_{1}^{m-1} z_{m}\right\|\right) .
\end{aligned}
$$

By induction on $M$, we have

$$
\begin{aligned}
& \left\|S_{M}^{m} S_{M-1}^{m} \ldots S_{1}^{m} z_{m}-S_{M}^{m-1} S_{M-1}^{m-1} \ldots S_{1}^{m-1} z_{m}\right\| \\
\leq & \left|\varpi_{m}^{1}-\varpi_{m-1}^{1}\right|\left(\left\|z_{m}\right\|+\left\|S_{1} z_{m}\right\|\right)+\left|\varpi_{m}^{2}-\varpi_{m-1}^{2}\right|\left(\left\|S_{1}^{m-1} z_{m}\right\|+\left\|S_{2} S_{1}^{m-1} z_{m}\right\|\right) \\
& +\ldots+\left|\varpi_{m}^{M}-\varpi_{m-1}^{M}\right|\left(\left\|S_{M-1}^{m-1} \ldots S_{1}^{m-1} z_{m}\right\|\right. \\
& \left.+\left\|S_{M} S_{M-1}^{m-1} \ldots S_{1}^{m-1} z_{m}\right\|\right) .
\end{aligned}
$$

Since $\lim _{m \rightarrow \infty}\left|\varpi_{m+1}^{j}-\varpi_{m}^{j}\right|=0$ for $j=1,2, \ldots, M$ and $\left\{z_{m}\right\},\left\{S z_{m}\right\},\left\{S_{1} z_{m}\right\}$ and $\left\|S_{1}^{m} z_{m}\right\|$ are all bounded, we get from condition (iv) that

$$
\lim _{m \rightarrow \infty}\left\|S_{M}^{m} S_{M-1}^{m} \ldots S_{1}^{m} z_{m}-S_{M}^{m-1} S_{M-1}^{m-1} \ldots S_{1}^{m-1} z_{m}\right\|=0 .
$$

Further, we evaluate

$$
\begin{aligned}
\left\|z_{m+1}-z_{m}\right\| & =\left\|y_{m+1}+\gamma A^{*}\left(w_{m+1}-A y_{m+1}\right)-y_{m}-\gamma A^{*}\left(w_{m}-A y_{m}\right)\right\| \\
& \leq\left\|y_{m+1}-y_{m}\right\|+\gamma\|A\|\left(\left\|w_{m+1}-w_{m}\right\|-\left\|A y_{m+1}-A y_{m}\right\|\right) .
\end{aligned}
$$

It follows that

$$
\begin{aligned}
\left\|y_{m+1}-y_{m}\right\|^{2} & =\left\|X\left(x_{m+1}\right)-X\left(x_{m}\right)\right\|^{2} \\
& =\left\|S_{r_{m}}^{F}\left(I-r_{m} f\right) x_{m+1}-S_{r_{m}}^{F}\left(I-r_{m} f\right) x_{m}\right\|^{2} \\
& \leq\left\|\left(x_{m+1}-x_{m}\right)-r_{m}\left(f x_{m+1}-f x_{m}\right)\right\|^{2} \\
& \leq\left\|x_{m+1}-x_{m}\right\|^{2}-r_{m}\left(2 \theta_{1}-r_{m}\right)\left\|f x_{m+1}-f x_{m}\right\|^{2} \\
& \leq\left\|x_{m+1}-x_{m}\right\|^{2}
\end{aligned}
$$

and

$$
\begin{aligned}
\left\|w_{m+1}-w_{m}\right\|^{2} & =\left\|Y\left(A y_{m+1}\right)-Y\left(A y_{m}\right)\right\|^{2} \\
& =\left\|S_{r_{m}}^{G}\left(I-r_{m} g\right) A y_{m+1}-S_{r_{m}}^{G}\left(I-r_{m} g\right) A y_{m}\right\|^{2} \\
& \leq\left\|A y_{m+1}-A y_{m}\right\|^{2}-r_{m}\left(2 \theta_{2}-r_{m}\right)\left\|g A y_{m+1}-g A y_{m}\right\|^{2} .
\end{aligned}
$$

From (3.3), we have

$$
\begin{aligned}
\left\|g A y_{m}-g A p\right\|^{2} & \leq\left[r_{m}\left(2 \theta_{2}-r_{m}\right)\right]^{-1}\left(\left\|A y_{m}-A p\right\|^{2}-\left\|w_{m}-A p\right\|^{2}\right) \\
& \leq\left[r_{m}\left(2 \theta_{2}-r_{m}\right)\right]^{-1}\left(\left\|A y_{m}-A p\right\|+\left\|w_{m}-A p\right\|\right)\left\|A y_{m}-w_{m}\right\| \\
& \leq 2\left[r_{m}\left(2 \theta_{2}-r_{m}\right)\right]^{-1}\|A\|\left\|y_{m}-p\right\|\left\|A y_{m}-w_{m}\right\| .
\end{aligned}
$$


From (3.4), we get

$$
\begin{aligned}
\gamma\left(1-\gamma\left\|A^{*}\right\|^{2}\right)\left\|w_{m}-A y_{m}\right\|^{2} & \leq\left\|x_{m}-p\right\|^{2}-\left\|z_{m}-p\right\|^{2} \\
& \leq\left\|x_{m}-z_{m}\right\|\left(\left\|x_{m}-p\right\|+\left\|z_{m}-p\right\|\right) \\
& \leq R_{1}\left\|x_{m}-z_{m}\right\| .
\end{aligned}
$$

where $R_{1}:=\sup _{m}\left\{\left\|x_{m}-p\right\|+\left\|z_{m}-p\right\|\right\}$. Note that

$$
\left\|x_{m}-z_{m}\right\| \leq\left\|x_{m}-y_{m}\right\|+\left\|y_{m}-z_{m}\right\| .
$$

Hence

$$
\begin{aligned}
\left\|z_{m}-p\right\|^{2}= & \left\|y_{m}+\gamma A^{*}\left(w_{m}-A y_{m}\right)-p\right\|^{2} \\
= & \left\langle y_{m}+\gamma A^{*}\left(w_{m}-A y_{m}\right)-p, z_{m}-p\right\rangle \\
= & \frac{1}{2}\left[\left\|\left(y_{m}-p\right)+\gamma A^{*}\left(w_{m}-A y_{m}\right)\right\|^{2}+\left\|z_{m}-p\right\|^{2}-\|\left(y_{m}-z_{m}\right)\right. \\
& \left.+\gamma A^{*}\left(w_{m}-A y_{m}\right) \|^{2}\right] \\
= & \frac{1}{2}\left[\left\|y_{m}-p\right\|^{2}+\left\|z_{m}-p\right\|^{2}+\left\|\gamma A^{*}\left(w_{m}-A y_{m}\right)\right\|^{2}\right. \\
& \left.+2 \gamma\left\langle A y_{m}-A p, w_{m}-A y_{m}\right\rangle-\left\|\left(z_{m}-y_{m}\right)+\gamma A^{*}\left(w_{m}-A y_{m}\right)\right\|^{2}\right] \\
\leq & \frac{1}{2}\left[\left\|y_{m}-p\right\|^{2}+\left\|z_{m}-p\right\|^{2}+\left\|\gamma A^{*}\left(w_{m}-A y_{m}\right)\right\|^{2}\right. \\
& +2 \gamma\left\|A y_{m}-A p\right\|\left\|w_{m}-A y_{m}\right\|-\left\|z_{m}-y_{m}\right\|^{2}-\left\|\gamma A^{*}\left(w_{m}-A y_{m}\right)\right\|^{2} \\
& \left.-2 \gamma\left\langle z_{m}-y_{m}, A^{*}\left(w_{m}-A y_{m}\right)\right\rangle\right] \\
\leq & \left\|y_{m}-p\right\|^{2}-\left\|z_{m}-y_{m}\right\|^{2}+2 \gamma\left\|A y_{m}-A p\right\|\left\|w_{m}-A y_{m}\right\| \\
& +2 \gamma\left\|z_{m}-y_{m}\right\|\left\|A^{*}\right\|\left\|w_{m}-A y_{m}\right\| \\
\leq & \left\|y_{m}-p\right\|^{2}-\left\|z_{m}-y_{m}\right\|^{2}+2 \gamma\left\|w_{m}-A y_{m}\right\|\left(\left\|A y_{m}-A p\right\|\right. \\
& \left.+\left\|A^{*}\right\|\left\|z_{m}-y_{m}\right\|\right)
\end{aligned}
$$

Using (3.2) and (3.4), we get

$$
\begin{aligned}
\left\|z_{m}-y_{m}\right\|^{2} \leq & \left\|y_{m}-p\right\|^{2}-\left\|z_{m}-p\right\|^{2}+2 \gamma\left\|A y_{m}-A p\right\|\left\|w_{m}-A y_{m}\right\| \\
& +2 \gamma\left\|z_{m}-y_{m}\right\|\left\|A^{*}\right\|\left\|w_{m}-A y_{m}\right\| \\
\leq & \left\|x_{m}-p\right\|^{2}-\left\|z_{m}-p\right\|^{2}+2 \gamma\left\|A y_{m}-A p\right\|\left\|w_{m}-A y_{m}\right\| \\
& +2 \gamma\left\|z_{m}-y_{m}\right\|\left\|A^{*}\right\|\left\|w_{m}-A y_{m}\right\| \\
\leq & 2 \gamma\left\|A y_{m}-A p\right\|\left\|w_{m}-A y_{m}\right\|+2 \gamma\left\|z_{m}-y_{m}\right\|\left\|A^{*}\right\|\left\|w_{m}-A y_{m}\right\| \\
\leq & 2 \gamma\left\|w_{m}-A y_{m}\right\|\left(\left\|A y_{m}-A p\right\|+\left\|A^{*}\right\|\left\|z_{m}-y_{m}\right\|\right) \\
\leq & 2 \gamma Q_{1}\left\|w_{m}-A y_{m}\right\|
\end{aligned}
$$

where $Q_{1}=\sup _{m}\left\{\left\|A y_{m}-A p\right\|+\left\|A^{*}\right\|\left\|z_{m}-y_{m}\right\|\right)$. Further, from (3.4), we have

$$
\begin{aligned}
\gamma\left(1-\gamma\left\|A^{*}\right\|^{2}\right)\left\|w_{m}-A y_{m}\right\|^{2} & \leq\left\|x_{m}-p\right\|^{2}-\left\|z_{m}-p\right\|^{2} \\
& \leq\left\|x_{m}-p\right\|^{2}-\left\|x_{m}-p\right\|^{2}=0 .
\end{aligned}
$$


Therefore,

$$
\lim _{m \rightarrow \infty}\left\|w_{m}-A y_{m}\right\|=0
$$

Hence from (3.13), we get

$$
\lim _{m \rightarrow \infty}\left\|z_{m}-y_{m}\right\|=0
$$

Next, we estimate

$$
\begin{aligned}
\left\|y_{m}-p\right\|^{2}= & \left\|X\left(x_{m}\right)-p\right\|^{2} \\
= & \left\|S_{r_{m}}^{F}\left(I-r_{m} f\right) x_{m}-S_{r_{m}}^{F}\left(I-r_{m} f\right) p\right\|^{2} \\
\leq & \left\langle\left(I-r_{m} f\right) x_{m}-\left(I-r_{m} f\right) p, y_{m}-p\right\rangle \\
= & \frac{1}{2}\left[\left\|\left(I-r_{m} f\right) x_{m}-\left(I-r_{m} f\right) p\right\|^{2}+\left\|y_{m}-p\right\|^{2}\right. \\
& \left.-\left\|x_{m}-y_{m}-r_{m}\left(f x_{m}-f p\right)\right\|^{2}\right] \\
\leq & \frac{1}{2}\left[\left\|x_{m}-p\right\|^{2}+\left\|y_{m}-p\right\|^{2}-\left\|x_{m}-y_{m}\right\|^{2}\right. \\
& \left.+2 r_{m}\left\langle x_{m}-y_{m}, f x_{m}-f p\right\rangle-r_{m}^{2}\left\|f x_{m}-f p\right\|^{2}\right] \\
\leq & \frac{1}{2}\left[\left\|x_{m}-p\right\|^{2}+\left\|y_{m}-p\right\|^{2}-\left\|x_{m}-y_{m}\right\|^{2}\right. \\
& \left.+2 r_{m}\left\|x_{m}-y_{m}\right\|\left\|f x_{m}-f p\right\|\right] \\
\leq & \left\|x_{m}-p\right\|^{2}-\left\|x_{m}-y_{m}\right\|^{2}+2 r_{m}\left\|x_{m}-y_{m}\right\|\left\|f x_{m}-f p\right\|
\end{aligned}
$$

From (3.2) and (3.16), we get

$$
\begin{aligned}
\left\|x_{m}-y_{m}\right\|^{2} & \leq\left\|x_{m}-p\right\|^{2}-\left\|y_{m}-p\right\|^{2}+2 r_{m}\left\|x_{m}-y_{m}\right\|\left\|f x_{m}-f p\right\| \\
& \leq 2 r_{m}\left\|x_{m}-y_{m}\right\|\left\|f x_{m}-f p\right\|
\end{aligned}
$$

Further, we have

$$
\left\|x_{m}-y_{m}\right\| \leq 2 r_{m}\left\|f x_{m}-f p\right\|
$$

From (3.2), we get

$$
r_{m}\left(2 \theta_{1}-r_{m}\right)\left\|f x_{m}-f p\right\|^{2} \leq\left\|x_{m}-p\right\|^{2}-\left\|y_{m}-p\right\|^{2}
$$

Therefore, we have $\lim _{m \rightarrow \infty}\left\|f x_{m}-f p\right\|=0$. From (3.17), we get

$$
\lim _{m \rightarrow \infty}\left\|x_{m}-y_{m}\right\|=0 .
$$

Suttituting (3.15) and (3.18) into (3.12), we get

$$
\lim _{m \rightarrow \infty}\left\|x_{m}-z_{m}\right\|=0 .
$$

From (3.7), we get

$$
\lim _{m \rightarrow \infty}\left\|g A y_{m+1}-g A y_{m}\right\|=0 .
$$

From (3.9) and (3.11), we get $\left\|z_{m+1}-z_{m}\right\| \leq\left\|y_{m+1}-y_{m}\right\|$ and $\left\|w_{m+1}-w_{m}\right\| \leq\left\|A y_{m+1}-A y_{m}\right\|$. From (3.10), we get $\left\|z_{m+1}-z_{m}\right\| \leq\left\|x_{m+1}-x_{m}\right\|$ From (3.5), (3.8) and using conditions (i-ii), we get

$$
\lim _{m \rightarrow \infty} \sup \left(\left\|w_{m+1}-w_{m}\right\|-\left\|x_{m+1}-x_{m}\right\|\right) \leq 0 .
$$

Using Lemma 2.7, we have

$$
\lim _{m \rightarrow \infty}\left\|w_{m}-x_{m}\right\|=0
$$


Further using condition (ii), we have

$$
\lim _{m \rightarrow \infty}\left\|x_{m+1}-x_{m}\right\|=\lim _{m \rightarrow \infty}\left(1-\beta_{m}\right)\left\|w_{m}-x_{m}\right\|=0 .
$$

Step 3. We show that

$$
\lim _{m \rightarrow \infty}\left\|x_{m}-S_{M}^{m} S_{M-1}^{m} \ldots S_{1}^{m} x_{m}\right\|=0
$$

From (3.1), we get

$$
\begin{aligned}
\left\|x_{m}-S_{M}^{m} S_{M-1}^{m} \ldots S_{1}^{m} x_{m}\right\| \leq & \left\|x_{m}-x_{m+1}\right\|+\left\|x_{m+1}-S_{M}^{m} S_{M-1}^{m} \ldots S_{1}^{m} x_{m}\right\| \\
\leq & \left\|x_{m}-x_{m+1}\right\|+\| \alpha_{m} \rho U\left(x_{m}\right)+\beta_{m} x_{m} \\
& +\left[\left(1-\beta_{m}\right) I-\alpha_{m} \mu V\right] S_{M}^{m} S_{M-1}^{m} \ldots S_{1}^{m} z_{m} \\
& -S_{M}^{m} S_{M-1}^{m} \ldots S_{1}^{m} x_{m} \| \\
\leq & \left\|x_{m}-x_{m+1}\right\|+\alpha_{m}\left\|\rho U\left(x_{m}\right)-\mu V\left(S_{M}^{m} S_{M-1}^{m} \ldots S_{1}^{m} z_{m}\right)\right\| \\
& +\beta_{m}\left\|x_{m}-S_{M}^{m} S_{M-1}^{m} \ldots S_{1}^{m} z_{m}\right\| \\
& +\left\|S_{M}^{m} S_{M-1}^{m} \ldots S_{1}^{m} z_{m}-S_{M}^{m} S_{M-1}^{m} \ldots S_{1}^{m} x_{m}\right\| \\
\leq & \left\|x_{m}-x_{m+1}\right\|+\alpha_{m}\left\|\rho U\left(x_{m}\right)-\mu V\left(S_{M}^{m} S_{M-1}^{m} \ldots S_{1}^{m} z_{m}\right)\right\| \\
& +\beta_{m}\left\|x_{m}-S_{M}^{m} S_{M-1}^{m} \ldots S_{1}^{m} z_{m}\right\|+\left\|z_{m}-x_{m}\right\|
\end{aligned}
$$

Next, we estimate

$$
\begin{aligned}
\left\|x_{m}-S_{M}^{m} S_{M-1}^{m} \ldots S_{1}^{m} z_{m}\right\| \leq & \left\|x_{m}-x_{m+1}\right\|+\left\|x_{m+1}-S_{M}^{m} S_{M-1}^{m} \ldots S_{1}^{m} z_{m}\right\| \\
\leq & \left\|x_{m}-x_{m+1}\right\|+\| \alpha_{m} \rho U\left(x_{m}\right)+\beta_{m} x_{m} \\
& +\left[\left(1-\beta_{m}\right) I-\alpha_{m} \mu V\right] S_{M}^{m} S_{M-1}^{m} \ldots S_{1}^{m} z_{m} \\
& -S_{M}^{m} S_{M-1}^{m} \ldots S_{1}^{m} z_{m} \| \\
\leq & \left\|x_{m}-x_{m+1}\right\|+\alpha_{m}\left\|\rho U\left(x_{m}\right)-\mu V\left(S_{M}^{m} S_{M-1}^{m} \ldots S_{1}^{m} z_{m}\right)\right\| \\
& +\beta_{m}\left\|x_{m}-S_{M}^{m} S_{M-1}^{m} \ldots S_{1}^{m} z_{m}\right\|
\end{aligned}
$$

Further, we have

$$
\begin{aligned}
\left\|x_{m}-S_{M}^{m} S_{M-1}^{m} \ldots S_{1}^{m} z_{m}\right\| \leq & \frac{1}{1-\beta_{m}}\left\|x_{m}-x_{m+1}\right\| \\
& +\frac{\alpha_{m}}{1-\beta_{m}}\left\|\rho U\left(x_{m}\right)-\mu V\left(S_{M}^{m} S_{M-1}^{m} \ldots S_{1}^{m} z_{m}\right)\right\|
\end{aligned}
$$

Using condition (i-ii), we have

$$
\lim _{m \rightarrow \infty}\left\|x_{m}-S_{M}^{m} S_{M-1}^{m} \ldots S_{1}^{m} z_{m}\right\|=0 .
$$

From (3.19), (3.20), (3.21) and using condition (i-ii), we have

$$
\lim _{m \rightarrow \infty}\left\|x_{m}-S_{M}^{m} S_{M-1}^{m} \ldots S_{1}^{m} x_{m}\right\|=0 .
$$

Step 4. We show that $\bar{p} \in \Gamma$.

First, we show that $\bar{p} \in \Omega$. Since $y_{m}=X\left(x_{m}\right)=S_{r_{m}}^{F}\left(I-r_{m} f\right) x_{m}$, we have

$$
F\left(y_{m}, q\right)+\left\langle f x_{m}, q-y_{m}\right\rangle+\frac{1}{r_{m}}\left\langle q-y_{m}, y_{m}-x_{m}\right\rangle \geq 0, \quad \forall q \in C .
$$


Since $F$ is monotone, we have

$$
\left\langle f x_{m}, q-y_{m}\right\rangle+\frac{1}{r_{m}}\left\langle q-y_{m}, y_{m}-x_{m}\right\rangle \geq F\left(q, y_{m}\right), \quad \forall q \in C .
$$

Hence replacing $m$ with $m_{v}$ in (3.23), we have

$$
\left\langle f x_{m_{v}}, q-y_{m_{v}}\right\rangle+\frac{1}{r_{m_{v}}}\left\langle q-y_{m_{v}}, y_{m_{v}}-x_{m_{v}}\right\rangle \geq F\left(q, y_{m_{v}}\right) \forall q \in C .
$$

Let $y_{i}=i y+(1-i) \bar{p} \in C$ with $0<i \leq 1$. So, from (3.24) we have

$$
\begin{aligned}
\left\langle y_{i}-y_{m_{v}}, f y_{i}\right\rangle \geq & \left\langle y_{i}-y_{m_{v}}, f y_{i}\right\rangle-\left\langle y_{i}-y_{m_{v}}, f x_{m_{v}}\right\rangle \\
& -\left\langle y_{i}-y_{m_{v}}, \frac{y_{m_{v}}-x_{m_{v}}}{r_{m_{v}}}\right\rangle+F\left(y_{i}, y_{m_{v}}\right) \\
= & \left\langle y_{i}-y_{m_{v}}, f y_{i}-f y_{m_{v}}\right\rangle+\left\langle y_{i}-y_{m_{v}}, f y_{m_{v}}-f x_{m_{v}}\right\rangle \\
& -\left\langle y_{i}-y_{m_{v}}, \frac{y_{m_{v}}-x_{m_{v}}}{r_{m_{v}}}\right\rangle+F\left(y_{i}, y_{m_{v}}\right)
\end{aligned}
$$

Since the sequences $\left\{x_{m}\right\},\left\{y_{m}\right\},\left\{z_{m}\right\}$ and $\left\{w_{m}\right\}$ have the same behaviour, so there exists subsequences $\left\{y_{m_{v}}\right\}$ of $\left\{y_{m}\right\},\left\{z_{m_{v}}\right\}$ of $\left\{z_{m}\right\},\left\{w_{m_{v}}\right\}$ of $\left\{w_{m}\right\}$ and $\left\{x_{m_{v}}\right\}$ of $\left\{x_{m}\right\}$ such that $z_{m_{v}} \rightarrow \bar{p}, w_{m_{v}} \rightarrow \bar{p}, x_{m_{v}} \rightarrow \bar{p}$ and $y_{m_{v}} \rightarrow \bar{p}$. Since $\lim _{v \rightarrow \infty}\left\|y_{m_{v}}-x_{m_{v}}\right\|=0$ and $f$ is lipschitz continuous, we have

$$
\lim _{v \rightarrow \infty}\left\|f y_{m_{v}}-f x_{m_{v}}\right\|=0 .
$$

Further since $\liminf \lim _{v \rightarrow \infty} r_{m_{v}}>0$, there exists a number $r>0$ such that $\liminf _{v \rightarrow \infty} r_{m_{v}}=r$. It follows that

$$
\begin{aligned}
\lim _{v \rightarrow \infty} \frac{\left\|y_{m_{v}}-x_{m_{v}}\right\|}{r_{m_{v}}} & \leq \frac{\lim _{v \rightarrow \infty}\left\|y_{m_{v}}-x_{m_{v}}\right\|}{\lim _{v \rightarrow \infty} \inf r_{m_{v}}} \\
& =\frac{1}{r} \lim _{v \rightarrow \infty}\left\|y_{m_{v}}-x_{m_{v}}\right\|=0 .
\end{aligned}
$$

From the monotonicity of $f$ and lower semicontinuity of $F$, we have from (3.25) that

$$
\left\langle y_{i}-\bar{p}, f y_{i}\right\rangle \geq F\left(y_{i}, \bar{p}\right) \text { as } v \rightarrow \infty
$$

and

$$
\begin{aligned}
0 & \leq F\left(y_{i}, y_{i}\right) \\
& \leq i F\left(y_{i}, q\right)+(1-i) F\left(y_{i}, \bar{p}\right) \\
& \leq i F\left(y_{i}, q\right)+(1-i)\left\langle y_{i}-\bar{p}, f y_{i}\right\rangle \\
& =i F\left(y_{i}, q\right)+(1-i) i\left\langle q-\bar{p}, f y_{i}\right\rangle .
\end{aligned}
$$

Hence,

$$
0 \leq F\left(y_{i}, q\right)+(1-i)\left\langle q-\bar{p}, f y_{i}\right\rangle
$$

Letting $i \rightarrow 0_{+}$, we have

$$
F(\bar{p}, q)+\langle q-\bar{p}, f \bar{p}\rangle \geq 0, \quad \forall q \in C .
$$

This implies that $\bar{p}$ solves problem (1.3). Since $A$ is bounded linear operator, we have $A y_{m_{v}} \rightarrow A \bar{p}$. Now setting $w_{m_{v}}=A y_{m_{v}}-Y A y_{m_{v}}$, it follows from (3.14) that $\lim _{v \rightarrow \infty} w_{m_{v}}=0$ and $A y_{m_{v}}-w_{m_{v}}=Y A y_{m_{v}}$. 
Therefore from Lemma 2.4, we have

$$
\begin{aligned}
& G\left(A y_{m_{v}}-w_{m_{v}}, r\right)+\left\langle g A y_{m_{v}}, r-\left(A y_{m_{v}}-w_{m_{v}}\right)\right\rangle \\
& +\frac{1}{r_{m_{v}}}\left\langle r-\left(A y_{m_{v}}-w_{m_{v}}\right), A y_{m_{v}}-w_{m_{v}}-A y_{m_{v}}\right\rangle \geq 0, \quad \forall r \in Q .
\end{aligned}
$$

Note that $G$ is upper semicontinuous in the first argument. Taking limsup in (3.26) as $v \rightarrow \infty$ and using $\liminf _{v \rightarrow \infty} r_{m_{v}}>0$, we get

$$
G(A \bar{p}, r)+\langle r-A \bar{p}, f A \bar{p}\rangle \geq 0, \quad \forall r \in Q .
$$

which implies that $\bar{q}=A \bar{p}$ solves problem (1.4) which shows that $\bar{p} \in \Omega$ and thus $\bar{p} \in \Gamma$.

Next we show that $\bar{p} \in \Phi$. Since $\left\{\varpi_{v}^{j}\right\}$ is bounded for $j=1,2, \ldots, M$, we assume that ${\varpi_{v}^{j}}^{j} \rightarrow{\varpi_{\infty}^{j}}^{j}$ as $t \rightarrow \infty$, where $0<\varpi_{\infty}^{j}<1$ for $j=1,2, \ldots, M$. Let $S_{j}^{\infty}=\left(1-\varpi_{\infty}^{j}\right) I+\varpi_{\infty}^{j} S_{j}$, for $j=1,2, \ldots, M$. Then

$$
\operatorname{Fix}\left(S_{j}^{\infty}\right)=\operatorname{Fix}\left(S_{j}\right) \text { for } j=1,2, \ldots, M \text {. }
$$

Since

$$
\begin{aligned}
\left\|S_{j}^{v_{t}} p-S_{j}^{\infty} p\right\| & =\left\|\left(1-\varpi_{v_{t}}^{j}\right) p+\varpi_{v_{t}}^{j} S_{j} p-\left(1-\varpi_{\infty}^{j}\right) p-\varpi_{\infty}^{j} S_{j} p\right\| \\
& \leq\left|\varpi_{v_{t}}^{j}-\varpi_{\infty}^{j}\right|\left(\|p\|+\left\|S_{j} p\right\|\right)
\end{aligned}
$$

we get

$$
\lim _{t \rightarrow \infty} \sup _{p \in D}\left\|S_{j}^{v_{t}} p-S_{j}^{\infty} p\right\|=0,
$$

where $D$ is an arbitrary bounded subset of $H_{1}$. Since $S_{j}^{\infty}$ is $\varpi_{\infty}^{j}$-averaged for $j=1,2, \ldots, M$, we have from Lemma 2.9 that $\bigcap_{j=1}^{M} F i x\left(S_{j}^{\infty}\right)=F i x\left(S_{1}^{\infty} S_{2}^{\infty} \ldots S_{M}^{\infty}\right)$. Since $\left\{x_{m}\right\}$ is bounded, there exists a subsequence $\left\{x_{m_{t}}\right\}$ of $\left\{x_{m}\right\}$ such that $x_{m_{t}} \rightarrow y$ as $t \rightarrow \infty$. Further, we have

$$
\begin{aligned}
\left\|x_{m_{t}}-S_{M}^{\infty} S_{M-1}^{\infty} \ldots S_{1}^{\infty} x_{m_{t}}\right\| \leq & \left\|x_{m_{t}}-S_{M}^{m_{t}} S_{M-1}^{m_{t}} \ldots S_{1}^{m_{t}} x_{m_{t}}\right\| \\
& +\left\|S_{M}^{m_{t}} S_{M-1}^{m_{t}} \ldots S_{1}^{m_{t}} x_{m_{t}}-S_{M}^{\infty} S_{M-1}^{\infty} \ldots S_{1}^{m_{t}} x_{m_{t}}\right\| \\
& +\left\|S_{M}^{\infty} S_{M-1}^{\infty} \ldots S_{1}^{m_{t}} x_{m_{t}}-S_{M}^{\infty} S_{M-1}^{\infty} \ldots S_{1}^{\infty} x_{m_{t}}\right\| \\
\leq & \left\|x_{m_{t}}-S_{M}^{m_{t}} S_{M-1}^{m_{t}} \ldots S_{1}^{m_{t}} x_{m_{t}}\right\| \\
& +\left\|S_{M}^{m_{t}} S_{M-1}^{m_{t}} \ldots S_{1}^{m_{t}} x_{m_{t}}-S_{M}^{\infty} S_{M-1}^{\infty} \ldots S_{1}^{m_{t}} x_{m_{t}}\right\| \\
& +\left\|S_{1}^{m_{t}} x_{m_{t}}-S_{1}^{\infty} x_{m_{t}}\right\| \\
\leq & \left\|x_{m_{t}}-S_{M}^{m_{t}} S_{M-1}^{m_{t}} \ldots S_{1}^{m_{t}} x_{m_{t}}\right\| \\
& +\sup _{p \in D^{\prime}}\left\|S_{M}^{m_{t}} p-S_{M}^{\infty} p\right\|+\sup _{p \in D^{\prime \prime}}\left\|S_{1}^{m_{t}} p-S_{1}^{\infty} p\right\|,
\end{aligned}
$$

where $D^{\prime}$ is a bounded subset including $\left\{S_{1}^{m_{t}} x_{m_{t}}\right\}$ and $D^{\prime \prime}$ is a bounded subset including $\left\{x_{m_{t}}\right\}$. From (3.22) and (3.27), we get

$$
\lim _{m \rightarrow \infty}\left\|x_{m_{t}}-S_{M}^{\infty} S_{M-1}^{\infty} \ldots S_{1}^{\infty} x_{m_{t}}\right\|=0 .
$$

From Lemma 2.2, we have $y \in \operatorname{Fix}\left(S_{M}^{\infty} S_{M-1}^{\infty} \ldots S_{1}^{\infty}\right)$.

Step 5. Finally, we claim that

$$
\lim _{m \rightarrow \infty} \sup \left\langle(\mu V-\rho Y) \bar{p}, \bar{p}-x_{m}\right\rangle \leq 0 .
$$


We have

$$
\begin{aligned}
\lim _{m \rightarrow \infty} \sup \left\langle(\mu V-\rho U) \bar{p}, \bar{p}-x_{m}\right\rangle & =\lim _{m \rightarrow \infty}\left\langle(\mu V-\rho U) \bar{p}, \bar{p}-x_{m_{v}}\right\rangle \\
& =\langle(\mu V-\rho U) \bar{p}, \bar{p}-y\rangle \\
& \leq 0 .
\end{aligned}
$$

Next, we show that $x_{m} \rightarrow \bar{p}$ as $m \rightarrow \infty$.

$$
\begin{aligned}
& \left\|x_{m+1}-\bar{p}\right\|^{2}=\| \alpha_{m}\left(\rho U\left(x_{m}\right)-\mu V(\bar{p})\right)+\beta_{m}\left(x_{m}-\bar{p}\right)+\left[\left(1-\beta_{m}\right) I-\alpha_{m} \mu V\right] S_{M}^{m} S_{M-1}^{m} \ldots S_{1}^{m} z_{m} \\
& -\left[\left(1-\beta_{m}\right) I-\alpha_{m} \mu V\right] S_{M}^{m} S_{M-1}^{m} \ldots S_{1}^{m} \bar{p} \|^{2} \\
& \leq \| \beta_{m}\left(x_{m}-\bar{p}\right)+\left[\left(1-\beta_{m}\right) I-\alpha_{m} \mu V\right] S_{M}^{m} S_{M-1}^{m} \ldots S_{1}^{m} z_{m} \\
& -\left[\left(1-\beta_{m}\right) I-\alpha_{m} \mu V\right] S_{M}^{m} S_{M-1}^{m} \ldots S_{1}^{m} \bar{p} \|^{2} \\
& +2 \alpha_{m}\left\langle\rho U\left(x_{m}\right)-\mu V(\bar{p}), x_{m+1}-\bar{p}\right\rangle \\
& \leq\left(\beta_{m}\left\|x_{m}-\bar{p}\right\|+\left(1-\beta_{m}\right) \|\left(I-\frac{\alpha_{m} \mu V}{1-\beta_{m}}\right) S_{M}^{m} S_{M-1}^{m} \ldots S_{1}^{m} z_{m}\right. \\
& -\left(I-\frac{\alpha_{m} \mu V}{1-\beta_{m}}\right) S_{M}^{m} S_{M-1}^{m} \ldots S_{1}^{m} \bar{p} \|^{2} \\
& +2 \alpha_{m} \rho\left\langle U\left(x_{m}\right)-U(\bar{q}), x_{m+1}-\bar{p}\right\rangle+2 \alpha_{m}\left\langle\rho U(\bar{p})-\mu V(\bar{p}), x_{m+1}-\bar{p}\right\rangle \\
& \leq\left(\beta_{m}\left\|x_{m}-\bar{p}\right\|+\left(1-\beta_{m}\right)\left(1-\frac{\alpha_{m} \zeta}{1-\beta_{m}}\right)\left\|z_{m}-\bar{p}\right\|\right)^{2} \\
& +2 \alpha_{m} \rho \sigma\left\|x_{m}-\bar{p}\right\|\left\|x_{m+1}-\bar{p}\right\|+2 \alpha_{m}\left\langle\rho U(\bar{p})-\mu V(\bar{p}), x_{m+1}-\bar{p}\right\rangle \\
& \leq\left(\beta_{m}\left\|x_{m}-\bar{p}\right\|+\left(1-\beta_{m}-\alpha_{m} \zeta\right)\left\|z_{m}-\bar{p}\right\|\right)^{2}+\alpha_{m} \rho \sigma\left(\left\|x_{m}-\bar{p}\right\|^{2}+\left\|x_{m+1}-\bar{p}\right\|^{2}\right) \\
& +2 \alpha_{m}\left\langle\rho U(\bar{p})-\mu V(\bar{p}), x_{m+1}-\bar{p}\right\rangle \\
& =\left[\left(1-\alpha_{m} \zeta\right)^{2}+\alpha_{m} \rho \sigma\right]\left\|x_{m}-\bar{p}\right\|^{2}+\alpha_{m} \rho \sigma\left\|x_{m+1}-\bar{p}\right\|^{2} \\
& +2 \alpha_{m}\left\langle\rho U(\bar{p})-\mu V(\bar{p}), x_{m+1}-\bar{p}\right\rangle \\
& \leq\left(\frac{\left(1-\alpha_{m} \zeta\right)^{2}+\alpha_{m} \rho \sigma}{1-\alpha_{m} \rho \sigma}\right)\left\|x_{m}-\bar{p}\right\|^{2} \\
& +\left(\frac{2 \alpha_{m}}{1-\alpha_{m} \rho \sigma}\right)\left\langle\rho U(\bar{p})-\mu V(\bar{p}), x_{m+1}-\bar{p}\right\rangle \\
& =\left(1-\frac{2(\zeta-\rho \sigma) \alpha_{m}}{1-\alpha_{m} \rho \sigma}\right)\left\|x_{m}-\bar{p}\right\|^{2}+\left(\frac{\alpha_{m}^{2} \zeta^{2}}{1-\alpha_{m} \rho \sigma}\right)\left\|x_{m}-\bar{p}\right\|^{2} \\
& +\left(\frac{2 \alpha_{m}^{2} \zeta^{2}}{1-\alpha_{m} \rho \sigma}\right)\left\|x_{m}-\bar{p}\right\|^{2}+\left(\frac{2 \alpha_{m}}{1-\alpha_{m} \rho \sigma}\right)\left\langle\rho U(\bar{p})-\mu V(\bar{p}), x_{m+1}-\bar{p}\right\rangle \\
& \leq\left(1-\frac{2(\zeta-\rho \sigma) \alpha_{m}}{1-\alpha_{m} \rho \sigma}\right)\left\|x_{m}-\bar{p}\right\|^{2}+\left(\frac{2 \alpha_{m}(\zeta-\rho \sigma)}{1-\alpha_{m} \rho \sigma}\right)\left\{\frac{\alpha_{m} \zeta^{2}}{2(\tau-\rho \sigma)} M_{1}\right. \\
& \left.+\left(\frac{1}{\zeta-\rho \sigma}\right)\left\langle\rho U(\bar{p})-\mu V(\bar{p}), x_{m+1}-\bar{p}\right\rangle\right\} \\
& =\left(1-\chi_{m}\right)\left\|x_{m}-\bar{p}\right\|^{2}+\chi_{m} \sigma_{m} \text {, }
\end{aligned}
$$


where $M_{1}=\sup \left\{\left\|x_{m}-\bar{p}\right\|^{2} \mid m \geq 0\right\}, \chi_{m}=\frac{2(\zeta-\rho \sigma) \alpha_{m}}{1-\alpha_{m} \rho \sigma}$ and

$$
\sigma_{m}=\frac{\alpha_{m} \zeta^{2}}{2(\zeta-\rho \sigma)} M_{1}+\frac{1}{\zeta-\rho \sigma}\left\langle\rho U(\bar{p})-\mu T(\bar{p}), x_{m+1}-\bar{p}\right\rangle
$$

Since $\chi_{m} \rightarrow 0, \sum_{m=0}^{\infty} \chi_{m}=\infty$ and $\limsup _{m \rightarrow \infty} \sigma_{m} \leq 0$. By applying Lemma 2.8, we get $x_{m} \rightarrow \bar{p}$ as $m \rightarrow$ $\infty$.

Now we give some results from Theorem 3.1. First, we give an iterative method to find the common solution of the HFPP (1.1) and the SEP (1.5)-(1.6).

Corollary 3.1. Let $H_{1}$ and $H_{2}$ be two real Hilbert spaces. Let $C \subseteq H_{1}$ and $Q \subseteq H_{2}$ be nonempty, closed and convex subsets. Let $A: H_{1} \rightarrow H_{2}$ be a bounded linear operator with its adjoint operator $A^{*}$. Let $F$ : $C \times C \rightarrow \mathbb{R}$ and $G: Q \times Q \rightarrow \mathbb{R}$ be bifunction satisfying Assumption 2.1 and let $G$ be upper semicontinuous and let $S_{j}: C \rightarrow C$ be a nonexpansive mapping for each $j=1,2, \ldots, M$. Let $V: C \rightarrow C$ be a l-lipschitzian continuous and $\eta$-strongly monotone mapping with $l>0$ and $\eta>0$ and let $U: C \rightarrow C$ be a $\tau$-lipschitzian continuous mapping with $\tau>0$. Let $0<\mu<\frac{2 \eta}{l^{2}}$ and $0<\rho \tau<\zeta$, where $\zeta=1-\sqrt{1-\mu\left(2 \eta-\mu l^{2}\right)}$. Assume that $\Gamma_{1}=\Omega_{1} \cap \Phi \neq \emptyset$. Let the iterative sequences $\left\{z_{m}\right\}$ and $\left\{x_{m}\right\}$ be generated by hybrid iterative algorithm:

$$
\left\{\begin{array}{l}
x_{0} \in C \\
z_{m}=S_{r_{m}}^{F}\left(x_{m}+\gamma A^{*}\left(S_{r_{m}}^{G}-I\right) A x_{m}\right) \\
x_{m+1}=\alpha_{m} \rho U\left(x_{m}\right)+\beta_{m} x_{m}+\left[\left(1-\beta_{m}\right) I-\alpha_{m} \mu V\right] S_{M}^{m} S_{M-1}^{m} \ldots S_{1}^{m} z_{m}, \forall m \geq 0
\end{array}\right.
$$

where $S_{j}^{m}=\left(1-\varpi_{m}^{j}\right) I+\varpi_{m}^{j} S_{j}, \gamma \in\left(0, \frac{1}{\|A\|^{2}}\right), \varpi_{m}^{j} \in\left(\xi_{1}, \xi_{2}\right)$ for some $\xi_{1}, \xi_{2} \in(0,1),\left\{\alpha_{m}\right\}$ and $\left\{\beta_{m}\right\}$ are two real sequences in $(0,1)$ and $\left\{r_{m}\right\} \subset(0, \infty)$ satisfy the conditions:

(1) $\lim _{m \rightarrow \infty} \alpha_{m}=0$ and $\sum_{m=1}^{\infty} \alpha_{m}=\infty$.

(2) $0<\liminf _{m \rightarrow \infty} \beta_{m} \leq \limsup _{m \rightarrow \infty} \beta_{m}<1$.

(3) $\lim _{m \rightarrow \infty} \inf r_{m}>0$.

(4) $\lim _{m \rightarrow \infty}\left|\varpi_{m+1}^{j}-\varpi_{m}^{j}\right|=0$ for $j=1,2, \ldots, M$.

Then the sequence $\left\{x_{m}\right\}$ converges strongly to $\bar{p} \in \Gamma_{1}$.

Proof. Setting $f=g=0$ in Theorem 3.1, we have the conclusion immediately.

Next, we give an iterative method to find a common solution of HFPP (1.1) and the SVIP (1.7)-(1.8).

Corollary 3.2. Let $H_{1}$ and $H_{2}$ be two real Hilbert spaces. Let $C \subseteq H_{1}$ and $Q \subseteq H_{2}$ be nonempty, closed and convex subsets. Let $A: H_{1} \rightarrow H_{2}$ be a bounded linear operator with its adjoint operator $A^{*}$. Let mapping $f: H_{1} \rightarrow H_{1}$ and $g: H_{2} \rightarrow H_{2}$ be $\theta_{1}$-inverse strongly monotone and $\theta_{2}$-inverse strongly monotone, respectively. Let $S_{j}: C \rightarrow C$ be a nonexpansive mapping for each $j=1,2, \ldots, M$. Let $V: C \rightarrow C$ be a l-lipschitzian continuous and $\eta$-strongly monotone mapping with $l>0$ and $\eta>0$ and let $U: C \rightarrow C$ be a $\tau$-lipschitzian continuous mapping with $\tau>0$. Let $0<\mu<\frac{2 \eta}{l^{2}}$ and $0<\rho \tau<\zeta$, where $\zeta=1-\sqrt{1-\mu\left(2 \eta-\mu k^{2}\right)}$. Assume that $\Gamma_{2}=\Omega_{2} \bigcap \Phi \neq \emptyset$. Let the iterative sequences $\left\{z_{m}\right\}$ and $\left\{x_{m}\right\}$ 
be generated by hybrid iterative algorithm:

$$
\left\{\begin{array}{l}
x_{0} \in C \\
y_{m}=X\left(x_{m}\right) ; w_{m}=Y\left(A y_{m}\right) \\
z_{m}=y_{m}+\gamma A^{*}\left(w_{m}-A y_{m}\right) \\
x_{m+1}=\alpha_{m} \rho U\left(x_{m}\right)+\beta_{m} x_{m}+\left[\left(1-\beta_{m}\right) I-\alpha_{m} \mu V\right] S_{M}^{m} S_{M-1}^{m} \ldots S_{1}^{m} z_{m}, \forall m \geq 0
\end{array}\right.
$$

where $S_{j}^{m}=\left(1-\varpi_{m}^{j}\right) I+\varpi_{m}^{j} S_{j}, X=P_{C}\left(I-r_{m} f\right), Y=P_{Q}\left(I-r_{m} g\right), \gamma \in\left(0, \frac{1}{\|A\|^{2}}\right), \varpi_{m}^{j} \in\left(\xi_{1}, \xi_{2}\right)$ for some $\xi_{1}, \xi_{2} \in(0,1),\left\{\alpha_{m}\right\}$ and $\left\{\beta_{m}\right\}$ are two real sequences in $(0,1)$ and $\left\{r_{m}\right\} \subset(0, \alpha)$, where $\alpha=$ $2 \min \left\{\theta_{1}, \theta_{2}\right\}$, satisfy the conditions:

(1) $\lim _{m \rightarrow \infty} \alpha_{m}=0$ and $\sum_{m=1}^{\infty} \alpha_{m}=\infty$.

(2) $0<\liminf _{m \rightarrow \infty} \beta_{m} \leq \limsup _{m \rightarrow \infty} \beta_{m}<1$.

(3) $\lim _{m \rightarrow \infty} \inf r_{m}>0$.

(4) $\lim _{m \rightarrow \infty}\left|\varpi_{m+1}^{j}-\varpi_{m}^{j}\right|=0$ for $j=1,2, \ldots, M$.

Then the sequence $\left\{x_{m}\right\}$ converges strongly to $\bar{p} \in \Gamma_{2}$.

Proof. Setting $F=G=0$, we get $S_{r_{m}}^{F}=P_{C}$ and $S_{r_{m}}^{G}=P_{Q}$ in Theorem 3.1.

Further, we give an iterative method to find a common solution of the HFPP (1.1) and the SMVIP (1.9)-(1.10).

Corollary 3.3. Let $H_{1}$ and $H_{2}$ be two real Hilbert spaces. Let $C \subseteq H_{1}$ and $Q \subseteq H_{2}$ be nonempty, closed and convex subsets. Let $A: H_{1} \rightarrow H_{2}$ be a bounded linear operator with its adjoint operator $A^{*}$. Let $N: H_{1} \rightarrow 2^{H_{1}}$ and $P: H_{2} \rightarrow 2^{H_{2}}$ be the multi-valued maximal monotone mappings. Let mappings $f: C \rightarrow$ $H_{1}$ and $g: Q \rightarrow H_{2}$ be $\theta_{1}$-inverse strongly monotone and $\theta_{2}$-inverse strongly monotone, respectively. Let $S_{j}: C \rightarrow C$ be a nonexpansive mapping for each $j=1,2, \ldots, M$. Let $V: C \rightarrow C$ be a l-lipschitzian continuous and $\eta$-strongly monotone mapping with $l>0$ and $\eta>0$ and let $U: C \rightarrow C$ be a $\tau$-lipschitzian continuous mapping with $\tau>0$. Let $0<\mu<\frac{2 \eta}{l^{2}}$ and $0<\rho \tau<\zeta$, where $\zeta=1-\sqrt{1-\mu\left(2 \eta-\mu l^{2}\right)}$. Assume that $\Gamma_{3}=\Omega_{3} \bigcap \Phi \neq \emptyset$. Let the iterative sequences $\left\{z_{m}\right\}$ and $\left\{x_{m}\right\}$ be generated by hybrid iterative algorithm:

$$
\left\{\begin{array}{l}
x_{0} \in C \\
y_{m}=X\left(x_{m}\right) ; w_{m}=Y\left(A y_{m}\right) \\
z_{m}=y_{m}+\gamma A^{*}\left(w_{m}-A y_{m}\right) \\
x_{m+1}=\alpha_{m} \rho U\left(x_{m}\right)+\beta_{m} x_{m}+\left[\left(1-\beta_{m}\right) I-\alpha_{m} \mu V\right] S_{M}^{m} S_{M-1}^{m} \ldots S_{1}^{m} z_{m}, \forall m \geq 0
\end{array}\right.
$$

where $S_{j}^{m}=\left(1-\varpi_{m}^{j}\right) I+\varpi_{m}^{j} S_{j}, X=J_{\lambda}^{N}(I-\lambda f), Y=J_{\lambda}^{P}(I-\lambda g), \gamma \in\left(0, \frac{1}{\|A\|^{2}}\right), \varpi_{m}^{j} \in\left(\xi_{1}, \xi_{2}\right)$ for some $\xi_{1}, \xi_{2} \in(0,1),\left\{\alpha_{m}\right\}$ and $\left\{\beta_{m}\right\}$ are two real sequences in $(0,1)$ and $\{\lambda\} \subset(0, \alpha)$, where $\alpha=2 \min \left\{\theta_{1}, \theta_{2}\right\}$, satisfy the conditions:

(1) $\lim _{m \rightarrow \infty} \alpha_{m}=0$ and $\sum_{m=1}^{\infty} \alpha_{m}=\infty$.

(2) $0<\liminf _{m \rightarrow \infty} \beta_{m} \leq \limsup \operatorname{sum}_{m \rightarrow \infty} \beta_{m}<1$.

(3) $\lim _{m \rightarrow \infty}\left|\varpi_{m+1}^{j}-\varpi_{m}^{j}\right|=0$ for $j=1,2, \ldots, M$.

Then the sequence $\left\{x_{m}\right\}$ converges strongly to $\bar{p} \in \Gamma_{3}$. 
Last, we give an iterative method to find a common solution of the HFPP (1.1) and the SNPP (1.11)(1.12).

Corollary 3.4. Let $H_{1}$ and $H_{2}$ be two real Hilbert spaces. Let $C \subseteq H_{1}$ and $Q \subseteq H_{2}$ be nonempty, closed and convex subsets. Let $A: H_{1} \rightarrow H_{2}$ be a bounded linear operator with its adjoint operator $A^{*}$. Let $N: H_{1} \rightarrow 2^{H_{1}}$ and $P: H_{2} \rightarrow 2^{H_{2}}$ be the multi-valued maximal monotone mappings and let $S_{j}: C \rightarrow C$ be a nonexpansive mapping for each $j=1,2, \ldots, M$. Let $V: C \rightarrow C$ be a l-lipschitzian continuous and $\eta$-strongly monotone mapping with $l>0$ and $\eta>0$ and let $U: C \rightarrow C$ be a $\tau$-lipschitzian continuous mapping with $\tau>0$. Let $0<\mu<\frac{2 \eta}{l^{2}}$ and $0<\rho \tau<\zeta$, where $\zeta=1-\sqrt{1-\mu\left(2 \eta-\mu l^{2}\right)}$. Assume that $\Gamma_{4}=\Omega_{4} \bigcap \Phi \neq \emptyset$. Let the iterative sequences $\left\{z_{m}\right\}$ and $\left\{x_{m}\right\}$ be generated by hybrid iterative algorithm:

$$
\left\{\begin{array}{l}
x_{0} \in C \\
z_{m}=J_{\lambda}^{N}\left(x_{m}+\gamma A^{*}\left(J_{\lambda}^{P}-I\right) A x_{m}\right) \\
x_{m+1}=\alpha_{m} \rho U\left(x_{m}\right)+\beta_{m} x_{m}+\left[\left(1-\beta_{m}\right) I-\alpha_{m} \mu V\right] S_{M}^{m} S_{M-1}^{m} \ldots S_{1}^{m} z_{m}, \forall m \geq 0
\end{array}\right.
$$

where $S_{j}^{m}=\left(1-\varpi_{m}^{j}\right) I+\varpi_{m}^{j} S_{j}, \gamma \in\left(0, \frac{1}{\|A\|^{2}}\right), \varpi_{m}^{j} \in\left(\xi_{1}, \xi_{2}\right)$ for some $\xi_{1}, \xi_{2} \in(0,1),\left\{\alpha_{m}\right\}$ and $\left\{\beta_{m}\right\}$ are two real sequences in $(0,1)$ and $\{\lambda\} \subset(0, \alpha)$, where $\alpha=2 \min \left\{\theta_{1}, \theta_{2}\right\}$, satisfy the conditions:

(1) $\lim _{m \rightarrow \infty} \alpha_{m}=0$ and $\sum_{m=1}^{\infty} \alpha_{m}=\infty$.

(2) $0<\liminf _{m \rightarrow \infty} \beta_{m} \leq \limsup _{m \rightarrow \infty} \beta_{m}<1$.

(3) $\lim _{m \rightarrow \infty}\left|\varpi_{m+1}^{j}-\varpi_{m}^{j}\right|=0$ for $j=1,2, \ldots, M$.

Then the sequence $\left\{x_{m}\right\}$ converges strongly to $\bar{p} \in \Gamma_{4}$.

\section{A NumericAl ExAMPLE}

Now we give a numerical example which illustrate Theorem 3.1.

Let $H_{1}=H_{2}=\mathbb{R}$, the set of all real numbers with the inner product defined by $\langle x, y\rangle=x y, \forall x, y \in \mathbb{R}$ and standard norm $|$.$| . Let C=[0,+\infty), Q=(-\infty, 0]$ and let $F: C \times C \rightarrow \mathbb{R}$ and $G: Q \times Q \rightarrow \mathbb{R}$ be defined by

$$
\begin{aligned}
& F(x, y)=x^{2}+y^{2}-3 x y, \quad \forall x, y \in C ; \\
& G(u, v)=2 u^{2}-3 v^{2}+5 u v, \quad \forall u, v \in Q
\end{aligned}
$$

Let the mappings $f: H_{1} \rightarrow H_{1}$ and $g: H_{2} \rightarrow H_{2}$ be defined by

$$
\begin{aligned}
& f(x)=x+2, \quad \forall x \in H_{1}, \\
& g(u)=5 u, \quad \forall u \in H_{2},
\end{aligned}
$$

respectively. Let the mapping $A: H_{1} \rightarrow H_{2}$ be defined by $A(x)=2 x, \forall x \in H_{1}$ and $S_{j}: C \rightarrow C, V: C \rightarrow C$ and $U: C \rightarrow C$ are defined by $S_{j} x=0$ for $j=1,2, \ldots, M, V(x)=2 x$ and $U(x)=\frac{x}{2}+1, \forall x \in C$. It is easy to see that $\varpi=\frac{1}{2}, \eta=l=2$. Hence $0<\mu<\frac{2 \eta}{l^{2}}=1$. Put $\mu=1$ we get $\zeta=1-\sqrt{1-\mu\left(2 \eta-\mu l^{2}\right)}=1$. From $0<\rho \tau<\zeta$, we have $0<\rho<2$, i.e., $\rho \in(0,2)$. Without loss of generality, we put $\rho=1$. Let

$$
\alpha_{m}=\frac{1}{3 m^{2}}, \beta_{m}=\frac{2 m-1}{3 m^{2}}, r_{m}=\frac{1}{2} \text { and } \varpi_{m}^{j}=\frac{1}{3}
$$


TABLE 1. Results for different initial values

\begin{tabular}{|l|l|l|l|}
\hline No. of iterations & $x_{m}$ & $x_{m}$ & $x_{m}$ \\
\hline & $x_{0}=-1.5$ & $x_{0}=1$ & $x_{0}=2$ \\
\hline $\mathrm{m}=1$ & -1.2540 & 0.5318 & 1.0637 \\
$\mathrm{~m}=5$ & -0.6937 & 0.2370 & 0.4741 \\
$\mathrm{~m}=10$ & -0.3556 & 0.1245 & 0.2489 \\
$\mathrm{~m}=15$ & -0.1867 & 0.0583 & 0.1165 \\
$\mathrm{~m}=20$ & -0.0874 & 0.0312 & 0.0623 \\
$\mathrm{~m}=25$ & -0.0467 & 0.0148 & 0.0295 \\
$\mathrm{~m}=30$ & -0.0222 & 0.0080 & 0.0159 \\
$\mathrm{~m}=35$ & -0.0119 & 0.0038 & 0.0076 \\
$\mathrm{~m}=40$ & -0.0057 & 0.0021 & 0.0041 \\
$\mathrm{~m}=45$ & -0.0031 & 0.0010 & 0.0020 \\
$\mathrm{~m}=50$ & -0.0015 & 0.0005 & 0.0011 \\
$\mathrm{~m}=55$ & -0.0009 & 0.0003 & 0.0005 \\
$\mathrm{~m}=60$ & -0.0004 & 0.0001 & 0.0003 \\
$\mathrm{~m}=65$ & -0.0002 & 0.0000 & 0.0001 \\
$\mathrm{~m}=70$ & -0.0000 & 0.0000 & 0.0000 \\
\hline
\end{tabular}

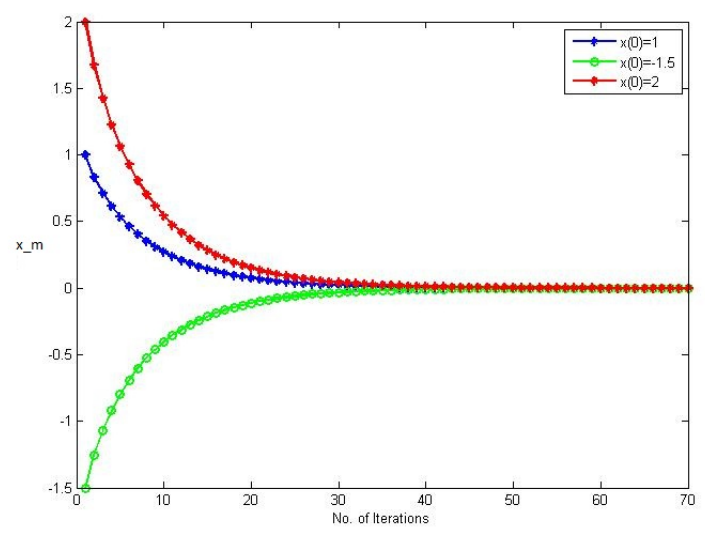

FIGURE 1. The convergence of $x_{m}$ with three initial values

for each $j=1,2, \ldots, M$. The sequences $\left\{\alpha_{m}\right\},\left\{\beta_{m}\right\},\left\{r_{m}\right\}$ and $\left\{\varpi_{m}^{j}\right\}$ satisfy conditions (i)-(iv). Since $S_{j} x=0$ for $j=1,2, \ldots, M$ and $A x=2 x$ for every $x \in \mathbb{R}$, we have

$$
\bigcap_{j=1}^{M} F i x\left(S_{j}\right)=\{0\}
$$

and $A$ is a bounded linear operator with $A^{*}=A$ and $\|A\|=2$ and hence $\gamma \in\left(0, \frac{1}{4}\right)$. Therefore, we choose $\gamma=0.2$. Further $f, g$ both are 1 and $\frac{1}{5}$ inverse strongly monotone mappings and hence $\left\{r_{m}\right\} \subset(0, \alpha)$, where $\alpha=2 \min \left\{1, \frac{1}{5}\right\}=\frac{2}{5}$. So we take $\lambda=\frac{1}{3}$, which yields that $\Phi=\operatorname{sol}(H F P P)=\{0\}$.

All codes were written in Matlab. The values of $\left\{x_{m}\right\}$ with different $m$ are given in Table 1. 


\section{CONCLUSION}

In this paper, we derived an iterative algorithm for finding a common solution of a split mixed equilibrium problem and a hierarchical fixed point problem for a finite family of nonexpansive mappings. We proved that the iterative algorithm converges strongly in Hilbert spaces. Finally, we presented a numerical example to clarify our main result. The method and results presented in this paper generalize and improve the corresponding results announced recently.

\section{REFERENCES}

[1] P.K. Anh, D.V. Hieu, Parallel hybrid iterative methods for variational inequalities, equilibrium problems, and common fixed point problems, Vietnam J. Math. 44 (2016), 351-374.

[2] H.H. Bauschke, P.L. Combettes, Convex Analysis and Monotone Operator Theory in Hilbert Spaces, Springer, New York, (2011).

[3] E. Blum, W. Oettli, From optimization and variational inequalities to equilibrium problems, Math. Stud. 63 (1994), 123145 .

[4] C. Byrne, Y. Gibali, S. Reich, The split common null point problem, J. Nonlinear Convex Anal. 13 (2012), 759-775.

[5] Y. Censor, A. Gibali, S. Reich, Algorithms for the split variational inequality problem, Numer. Algo. 59 (2012), $301-323$.

[6] S.Y. Cho, Strong convergence analysis of a hybrid algorithm for nonlinear operators in a Banach space, J. Appl. Anal. Comput. 8 (2018), 19-31.

[7] S.Y. Cho, B.A. Bin Dehaish, X. Qin, Weak convergence of a splitting algorithm in Hilbert spaces J. Appl. Anal. Comput. 7 (2017), 427-438.

[8] P.L. Combettes, S.A. Hirstoaga, Equilibrium programming using proximal like algorithms, Math. Program. 78 (1997), 29-41.

[9] K. Goebel, W. A. Kirk, Topics in Metric Fixed Point Theory, Stud. Adv. Math. Vol. 28, Cambridge University Press, Cambridge, 1990.

[10] S. He, L. Liu, X. Qin, A self-adaptive hybrid steepest descent algorithm for solving a class of variational inequalities, J. Nonlinear Funct. Anal. 2018 (2018), Article ID 49.

[11] S. Husain, N. Singh, Hybrid steepest iterative algorithm for a hierarchical fixed point problem, Fixed Point Theory Appl. 2017 (2017), Article ID 25.

[12] Jae Ug Jeong, Nonlinear algorithms for a common solution of a system of variational inequalities, a split equilibrium problem and fixed point problems, Korean J. Math. 24 (2016), 495-524.

[13] K.R. Kazmi, R.Ali, M. Furkan, Hybrid iterative method for split monotone variational incclusion problem and hierarcchical fixed point problem for a finite family of nonexpansive mappings, Numer. Algor. 79 (2018), 499-527.

[14] K.R. Kazmi, R.Ali, M. Furkan, Krasnoselski-Mann type iterative method for hierarcchical fixed point problem and split mixed equilibrium problem, Numer. Algor. 77 (2018), 289-308.

[15] K.R. Kazmi, S.H. Rizvi, Iterative approximation of a common solution of a split equilibrium problem, a variational inequality problem and a fixed point problem, J. Egyptian Math. Soc. 21 (2013), 44-51.

[16] L. Liu, A hybrid steepest descent method for solving split feasibility problems involving nonexpansive mappings, J Nonlinear Convex Anal. 20 (2019), 471-488.

[17] A. Moudafi, P. E. Maingé, Towards viscosity approximations of hierarchical fixed-point problems, Fixed Point Theory Appl. 2006 (2006), Article ID 95453.

[18] A. Moudafi, Split monotone variational inclusions, J. Optim. Theory Appl. 150 (2011), 275-283.

[19] X. Qin, Y.J. Cho, S.M. Kang, Convergence theorems of common elements for equilibrium problems and fixed point problems in Banach spaces, J. Comput. Appl. Math. 225 (2009), 20-30.

[20] N. Shahzad, H. Zegeye, Convergence theorems of common solutions for fixed point, variational inequality and equilibrium problems, J. Nonlinear Var. Anal. 3 (2019), 189-203.

[21] T. Suzuki, Strong convergence of Krasnoselskii and Mann's type sequences for one parameter nonexpansive semigroups without Bochner integrals, J. Math. Anal. Appl. 35 (2005), 227-239. 
[22] X. Zhi, H. Zhou, Y.J. Cho, Iterative solutions of nonlinear equations for m-accretive operators in Banach spaces, J. Nonlinear Convex Anal. 1 (2000), 313-320.

[23] I. Yamada, The hybrid steepest descent method for the variational inequality problems over the intersection of fixed points sets of nonexpansive mapping. In: D. Butnariu, Y. Censor, S. Reich (eds). Inherently Parallel Algorithms in Feasibility and Optimization and Their Application, pp. 473-504, North-Holland, Amsterdam, 2001.

[24] C. Zhang, C. Yang, A new explicit iterative algorithm for solving a class of variational inequalities over the common fixed points set of a finite family of nonexpansive mappings, Fixed Point Theory Appl. 2014 (2014), Article ID 60. 